PALEO

Revue d'archéologie préhistorique

13 | 2001

Varia

\title{
Étude préliminaire des cerfs du gisement pléistocène inférieur de Ceyssaguet (Haute-Loire)
}

Preliminary data on fossil deer from inferior Pleistocene of Ceyssaguet (HauteLoire)

Roman Croitor et Marie-Francoise Bonifay

\section{CpenEdition}

Journals

Édition électronique

URL : http://journals.openedition.org/paleo/1011

DOI : 10.4000/paleo.1011

ISSN : 2101-0420

Éditeur

SAMRA

Édition imprimée

Date de publication : 14 décembre 2001

Pagination : 129-144

ISSN : 1145-3370

Référence électronique

Roman Croitor et Marie-Francoise Bonifay, "Étude préliminaire des cerfs du gisement pléistocène inférieur de Ceyssaguet (Haute-Loire) », PALEO [En ligne], 13 | 2001, mis en ligne le 26 mai 2010, consulté le 07 juillet 2020. URL : http://journals.openedition.org/paleo/1011 ; DOI : https://doi.org/ 10.4000/paleo.1011

Ce document a été généré automatiquement le 7 juillet 2020

\section{(c) (i) (9)}

PALEO est mis à disposition selon les termes de la licence Creative Commons Attribution - Pas d'Utilisation Commerciale - Pas de Modification 4.0 International. 


\title{
Étude préliminaire des cerfs du gisement pléistocène inférieur de Ceyssaguet (Haute-Loire)
}

\author{
Preliminary data on fossil deer from inferior Pleistocene of Ceyssaguet (Haute- \\ Loire)
}

Roman Croitor et Marie-Francoise Bonifay

1 Le site de Ceyssaguet (Haute Loire) est anciennement connu par des ramassages de surface et les fossiles recueillis font partie des collections du Musée National d'Histoire Naturelle (Paris); le gisement est cité pour la première fois dans des travaux parus à la fin du siècle dernier (M. Boule, 1889) et l'étude ne porte que sur trois demi-mandibules de Canis.

2 Les fouilles entreprises sous la responsabilité de l'un d'entre nous (MF. B.) entre 1982 et 1997, ont permis d'exhumer une masse importante de fossiles (plus de 15000), tous répertoriés avec leurs coordonnées et systématiquement portés sur plan. En fait, le gisement de Ceyssaguet est actuellement un des très rares sites ayant livré des faunes villafranchiennes terminales qui ait été fouillé de façon moderne (MF. Bonifay, 1991). Depuis fin 1997, les faunes sont à l'étude et peu à peu apparaît une image de plus en plus nette de l'environnement contemporain liée à une meilleure connaissance des différents groupes.

\section{Aperçu sur la géologie et la chronologie du site}

3 En Europe, les sites villafranchiens sont groupés principalement sur les régions sud: Italie, Espagne, sud de la Russie, plus exceptionnellement et ponctuellement sud de l'Angleterre et de la Hollande. Les faunes contemporaines du déclin de cet ensemble biostratigraphique sont rares: en Italie, où les paléontologues les ont initialement reconnues, il n'y a jamais eu de fouilles systématiques et en Espagne, seuls un ou deux niveaux correspondent à cette période (MF. Bonifay, 1995) . 
4 Le gisement de Ceyssaguet (fig. 1) est situé dans le Massif central français sur la pente extérieure d'un volcan dont la dernière explosion a été datée par Potassium/Argon de 1.3 Ma par le Laboratoire de Tephrochronologie de la Faculté des Sciences de ClermontFerrand; cet âge absolu le situe à la fin de l'épisode biostratigraphique des faunes villafranchiennes qui sont connues en Velay depuis $2.4 \mathrm{Ma}$. Pour des raisons stratigraphiques - les faunes n'étant pas déposées directement sur le basalte daté - on peut estimer l'âge des niveaux fossilifères à 1.2 Ma. L'association animale représentée est une association villafranchienne que l'on pouvait croire, jusqu'à cette étude, relativement pauvre en espèces d'Artiodactyles.

5 Le sédiment des niveaux fossilifères est un loess dégradé dont le dépôt s'est effectué dans un sillon peu à peu élargi en gorge par l'eau s'échappant du lac de cratère.

Figure 1 - Le site de Ceyssaguet : les loess en cours de fouilles. Figure 1 - The site of Ceyssaguet: excavations in loessic sediment.

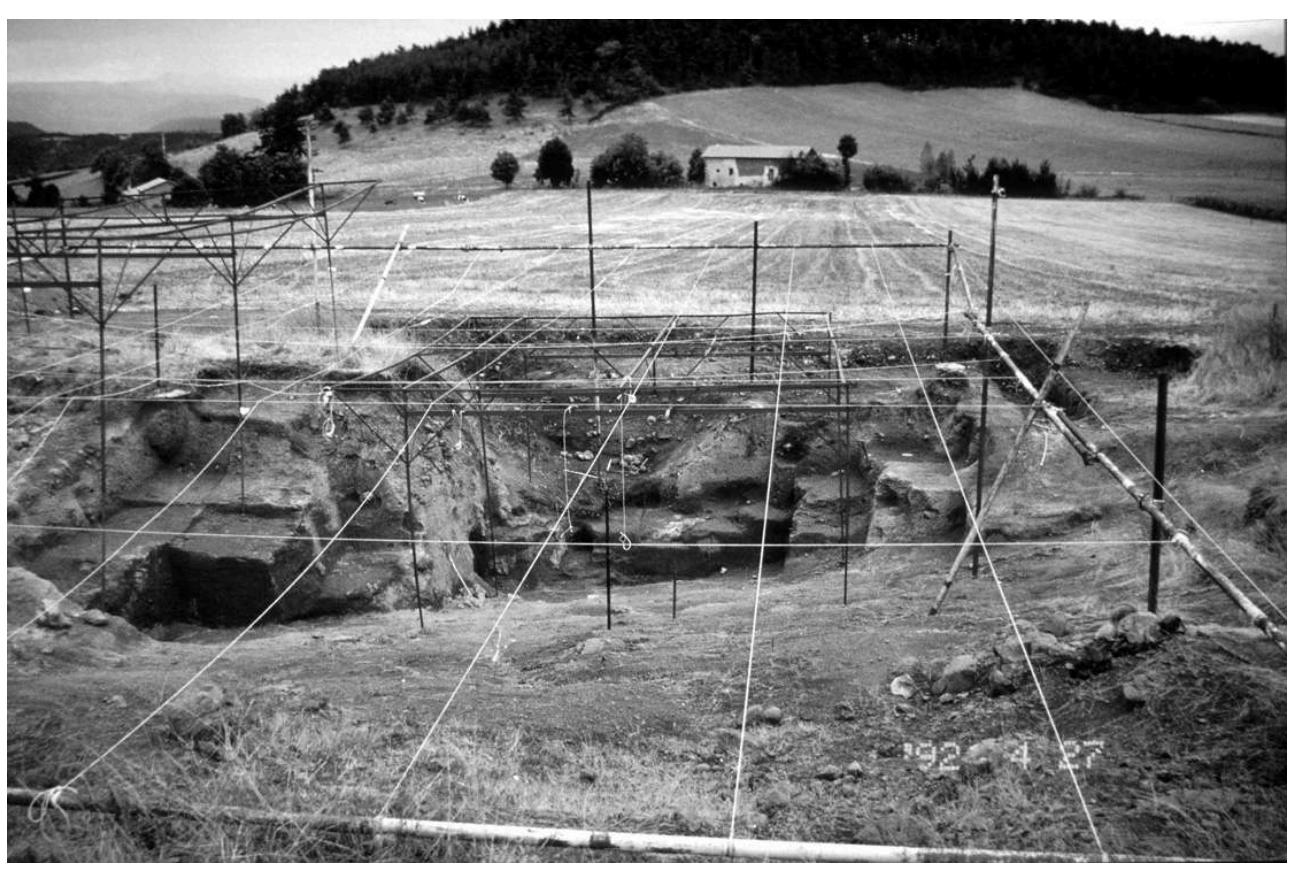

Les loess sont des sédiments très particuliers caractéristiques de climats assez froids mais surtout secs et venteux, dont l'origine se trouve dans les grandes plaines sans végétation du nord de l'Europe. Ils se sont déposés sur le flanc du volcan après la rupture de pente formée par le sommet du cratère actuellement érodé.

Ceyssaguet est le seul site européen, avec certains niveaux de Saint Vallier (Drôme) d'âge plus ancien, a fournir desfaunes villafranchiennes contemporaines d'un environnement loessique.

L'association villafranchienne représentée à Ceyssaguet est riche en espèces mais elle dominée par deux populations, celle des Cervidés et celle des Chevaux.

Les Carnivores comme les restes d'éléphants sont rares; les premiers sont qualitativement bien représentés puisque la majorité des espèces villafranchiennes sont présentes : le loup archaïque (Canis etruscus), un Renard, l'Hyène (Crocuta perrieri), le Lynx (Lynx issiodorensis), le Guépard (Acinonyx), un Machairodonte et deux Ours dont l'un est Ursus etruscus : ces restes peu abondants, sont en cours d'étude. 
10 La plupart des vestiges d'Eléphants appartiennent à l'éléphant méridional mais il est vraisemblable qu'il y ait aussi des restes de Loxodonta (N. Aouadi et MF. Bonifay, 1998). Les Chevaux sont plus abondants dans la partie supérieure des loess où ils sont accompagnés par des restes de Rhinoceros (Dicerorhinus etruscus ); les études récentes ont mis en évidence la présence de deux espèces d'Equidés: Equus stenonis et Equus bressanus (N. Aouadi, 1999).

La population de Cervidés que l'on pouvait penser pauvre en espèces se révèle après étude, beaucoup plus diversifiée que cela n'apparaissait à la fouille. L'étude préliminaire présentée ici montre qu'elle est en réalité composée de quatre espèces qui se regroupent en deux catégories : celle des cerfs de grande taille et celle des cerfs de taille moyenne. Globalement, les restes de Cervidés sont plus nombreux dans la partie inférieure des loess où ils se présentent très souvent en connexion anatomique.

Enfin, il faut souligner que ces faunes villafranchiennes ont l'intérêt d'être contemporaines des premiers peuplements humains européens. Dans le cas de Ceyssaguet, la fouille met en évidence deux niveaux taphonomiquement très différents :

- le niveau supérieur des loess livre des ossements fracturés, cassés, pratiquement jamais en connexion anatomique et dont la représentation numérique correspond à un tri (M.F.

Bonifay,1986)

- le niveau inférieur séparé du précédent par un sol très altéré, mince et mal visible, est de toute évidence d'origine naturelle avec une grande partie des ossements en connexion anatomique et une très nette dominance des Cervidés.

L'opposition entre l'organisation taphonomique des parties supérieure et inférieure des loess a l'intérêt d'avoir été mise en évidence dans un même sédiment (ici le loess); ceci permet d'annuler les biais dus à une conservation différentielle initiée par une sédimentation différente et donne une certaine unité à l'étude du site.

Il est évident que les niveaux supérieurs présentent un grand intérêt taphonomique : on peut remarquer que les os longs présentent des fractures dîtes "sur os frais" en milieu de diaphyse avec conservation des épiphyses (exemple des tibias de Chevaux : F. Baratta, 1998) et que la représentation du squelette est déséquilibrée (manque de vertèbres et de phalanges, abondance de bois de chute de cerfs non rongés), ce qui correspond bien à ce que l'on peut trouver sur un site de prédation. Le travail en cours consiste à identifier le prédateur.

\section{Étude systématique des Cervidés}

\section{1 - Données sur la systématique des Cerfs de grande taille}

Les Cervidés de grande taille des genres Eucladoceros et Praemegaceros appartiennent à des lignées qui se développent en Europe durant le Villafranchien et le Galérien (Azzarolli et Mazza, 1992, 1993). Ce groupe est bien connu car il a fourni de nombreux fossiles correspondant à différentes espèces présentant des formes de transition.

Cependant, la systématique des Cerfs de grande taille est plutôt confuse en partie parce que les descriptions anatomiques des genres et des espèces sont incomplètes et d'autre part à cause des nombreuses synonymies tant générique que spécifique. C'est la raison pour laquelle quelques questions de nomenclature et de systématique sont discutées 
dans ce travail afin d'aider à clarifier la position systématique des cerfs découverts à Ceyssaguet.

Une première précision doit être apportée au niveau du nom générique.

\section{Le genre Praemegaceros}

19 L'espèce type, Megaceroides algericus Lydekker, 1890 est considérée comme une branche spécialisée du groupe verticornis qui a survécu isolée au Pléistocène tardif en Afrique du Nord. Plus tard, Azzarolli (1979) défend le point de vue d'Ambrosetti (1967) lorsqu'il considère comme correct le nom de genre Megaceroides créé par Lydekker pour le “ verticornis group".

Puis Azzarolli et Mazza (1993) suggèrent que M. algericus est une espèce de taille réduite car ils mettent en évidence la disproportion entre les fortes meules et un merrain plus frêle, ce qui rappelle M. dawkinsi. Le front aplati et même convexe du Cerf africain est considéré ici comme un caractère important qui s'oppose au front concave du genre Megaloceros.

21 Hadjiouis (1990) revoit la position systématique de Megaceroides algericus et met en évidence sa ressemblance morphologique avec Sinomegaceros pachiosteus d'Asie. Un caractère très important est la morphologie de la mandibule et de la dentition : outre la présence d'un cingulum puissant sur les molaires supérieures, une faible longueur des prémolaires inférieures, un museau court et brachyodonte rapprochent le cerf algérien de Megaloceros giganteusl. Ainsi la morphologie dentaire et certaines proportions crâniennes suggèrent que le cerf d'Algérie est phylogénétiquement proche et de Megaloceros giganteus et des Sinomegaceros asiatiques.

En 1956, Kahlke qui travaillait sur les cerfs géants quaternaires d'Allemagne proposa le nouveau nom générique d'Orthogonoceros pour l'espèce-type Cervus verticornis Dawkins ; il le remplaça par la suite par le nom de genre Praemegaceros créé auparavant en 1920 par Portis (Kahlke, 1965).

23 Enfin, Radulesco et Samson (1967), puis Lister (1993) proposent que le nom de genre Praemegaceros ne corresponde qu'aux cerfs géants du groupe "verticornis". C'est cette dernière option que nous suivrons dans ce travail.

\section{Le genre Eucladoceros}

24 Le problème principal de la systématique du genre Eucladoceros est qu'il comporte une longue liste d'espèces ( plus de 14) qui ne sont pas clairement définies. Ainsi, bien que le nombre de fossiles de ce groupe soit plutôt important, il manque les descriptions morphologiques du crâne et des dentitions, les espèces d'Eucladoceros se distinguent pour la plupart par des caractères apomorphes dans la morphologie des bois.

Heintz (1970) reconnaît sept espèces correspondant à ce genre : outre Eucladoceros boulei asiatique, six espèces européennes: E. sedgwicki, E. ctenoides, E. dicranios, E. tegulensis, E. senezensis E. tertraceros. Vislobokova (1990) rapporte à ce genre Cervus ernesti Fritsch 1884 et Psecupsoceros orientalis Radulesco et Samson 1967. Dans de nombreux cas la fragmentation du matériel empêche la vérification des critères spécifiques.

De Vos et al. (1995) après révision du genre ne reconnaissent que trois espèces : $E$. dicranios aux andouillers dichotomiques, E. ctenoides et E. tetraceros aux bois à courbure 
simple. Pour ces deux derniers taxons qui ne se distinguent que par le degré de courbure des andouillers ou la distance entre les andouillers, les variations peuvent très bien être d'ordre intraspécifique et $E$. tetraceros peut alors être considéré comme une unité systématique de rang sous spécifique d'Eucladoceros ctenoides.

La nouvelle espèce E. giulii récemment créée par Kahlke (1997) est un autre Cerf problématique allemand: le spécimen décrit par l'auteur présente une courbure simple des bois ce qui permet de penser qu'il pourra peut-être par la suite être considéré comme un synonyme d'E.ctenoides.

La conclusion définitive ne pourra être proposée qu'après révision de tout le matériel d' Eucladoceros du groupe ctenoides- tetraceros. Dans le travail qui suit nous utiliserons la dénomination Eucladoceros ctenoides Nesti 1841 pour les Cerfs présentant une construction de bois à courbure simple.

\section{2 - Description anatomique des Cerfs de grande taille de Ceyssaguet}

A Ceyssaguet, le plus grand nombre de restes de cerfs appartient à E. ctenoides. Cette espèce est représentée par tout le squelette: crâne, bois, mandibules et restes postcrâniens. Quelques os des membres ont été trouvés in situ en connexion anatomique.

Il est intéressant de constater que les bois trouvés sont des bois de chute ce qui permet de penser qu'E. ctenoides n'était présent sur le site qu'à la période de perte des bois (printemps); d'autre part, la grande quantité de restes d'Eucladoceros trouvés tend à prouver qu'il s'agissait d'une espèce grégaire.

\section{1 - Genre Eucladoceros Falconer | Eucladoceros ctenoides Nesti,1841}

31 Synonymies :

Eucladoceros giuli sp.nov. Kahlke,1997 (p.225, tab. 40, fig. 1 - 6)

Matériel étudié dans le cadre de l'étude préliminaire :

Autre : fémur gauche (12581), tibia gauche $(2 / 3,10876)$

Le crâne 2 - 7217 appartient à un jeune adulte mâle avec une dentition complète et de longs et fins pédicules. En avant des pédicules, les os frontaux sont larges et concaves; la suture frontale forme une ride osseuse nette qui commence au niveau du milieu des arcades. Quelques différences morphologiques peuvent être relevées sur le crâne de Ceyssaguet par rapport à celui de l'espèce-type Eucladoceros dicranios : contrairement au spécimen italien, le crâne étudié est plus long et plus étroit au niveau des arcades zygomatiques, il en est de même du neurocrâne. La divergence entre l'axe longitudinal du neurocrâne et celui de la face est plus courte, ce qui entraîne une boite crânienne sans flexion, caractère considéré comme primitif par Vislobokova (1990). La rangée dentaire est comparativement plus longue ; le foramen ovale est grand: son plus grand diamètre est de $19.6 \mathrm{~mm}$, le diamètre transverse mesure $14.8 \mathrm{~mm}$.

Le crâne de femelle (2 - 10590) partiellement conservé présente des fosses préorbitaires petites et peu profondes (fig. 2). 
Figure 2 - Crâne d'Eucladoceros ctenoides femelle (2 - 10590). Figure 2 - Cranium of female Eucladoceros ctenoides (2 - 10590).

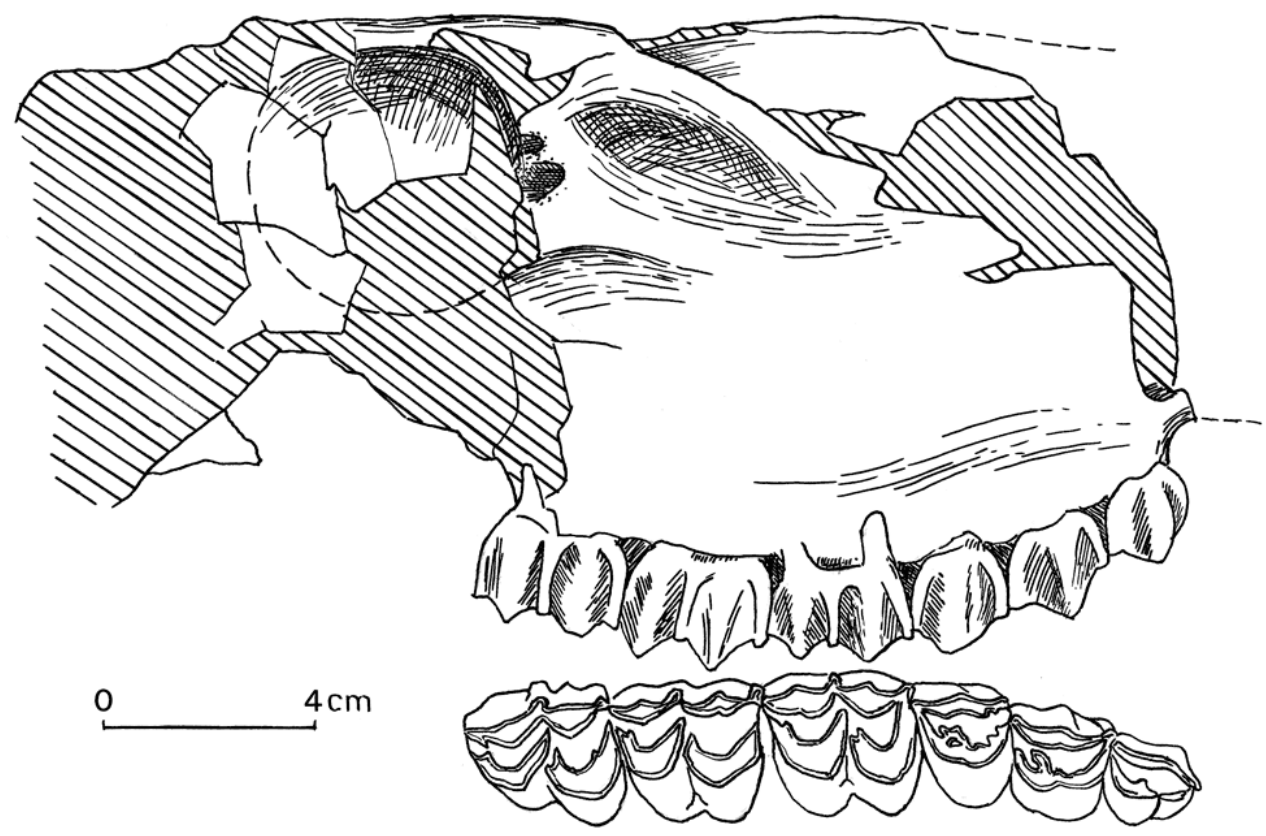

Les bois : La construction générale des bois est typique du groupe Eucladoceros. Perche et andouillers sont comprimés latéralement. Le nombre d'andouillers est supérieur à six et les andouillers terminaux sont souvent munis de pointes surnuméraires (2 1442). Tous les andouillers sont à peu près situés dans le même plan. En comparant la population de Cerfs de Ceyssaguet avec les holotypes d'Eucladoceros tetraceros et E. ctenoides, on peut observer une différence morphologique mineure sur le premier segment de perche qui est comprimé latéralement sur les bois de Ceyssaguet; chez $E$. tetraceros cette portion de bois est arrondie et chez E. ctenoides elle est comprimée dorso-ventralement, ce qui rappelle d'ailleurs la morphologie des Praemegaceros primitifs (fig. 3 et 4 ). 
Figure 3 - Bois de chute d'Eucladoceros ctenoides ( 2 - 3331).

Figure 3 - Shed antler of Eucladoceros ctenoides (2 - 3331).

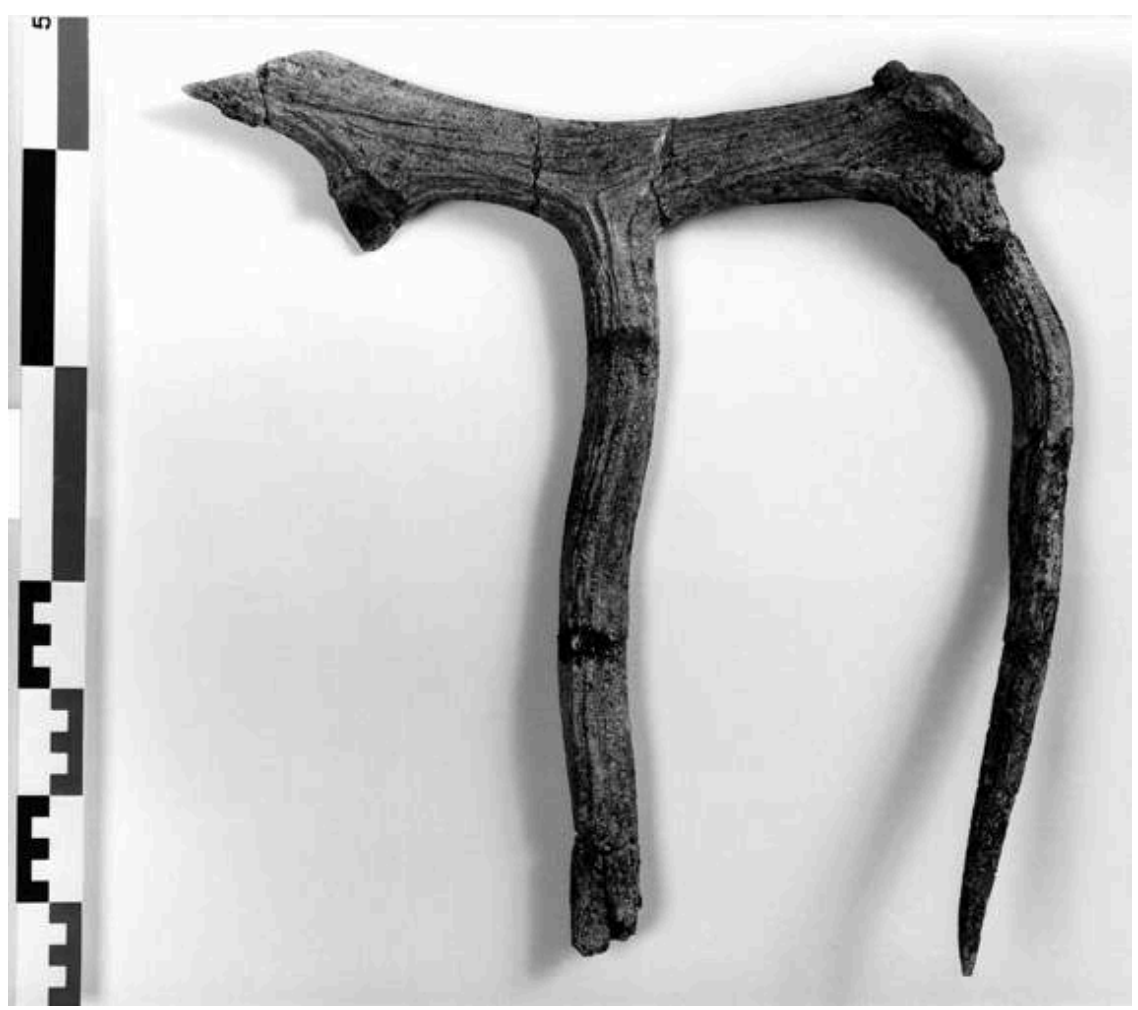

Figure 4 - Bois de chute in situ d'Eucladoceros ctenoides ( 2 - 1442). Figure 4 - Shed antler in situ of Eucladoceros ctenoides ( 2 - 1442).

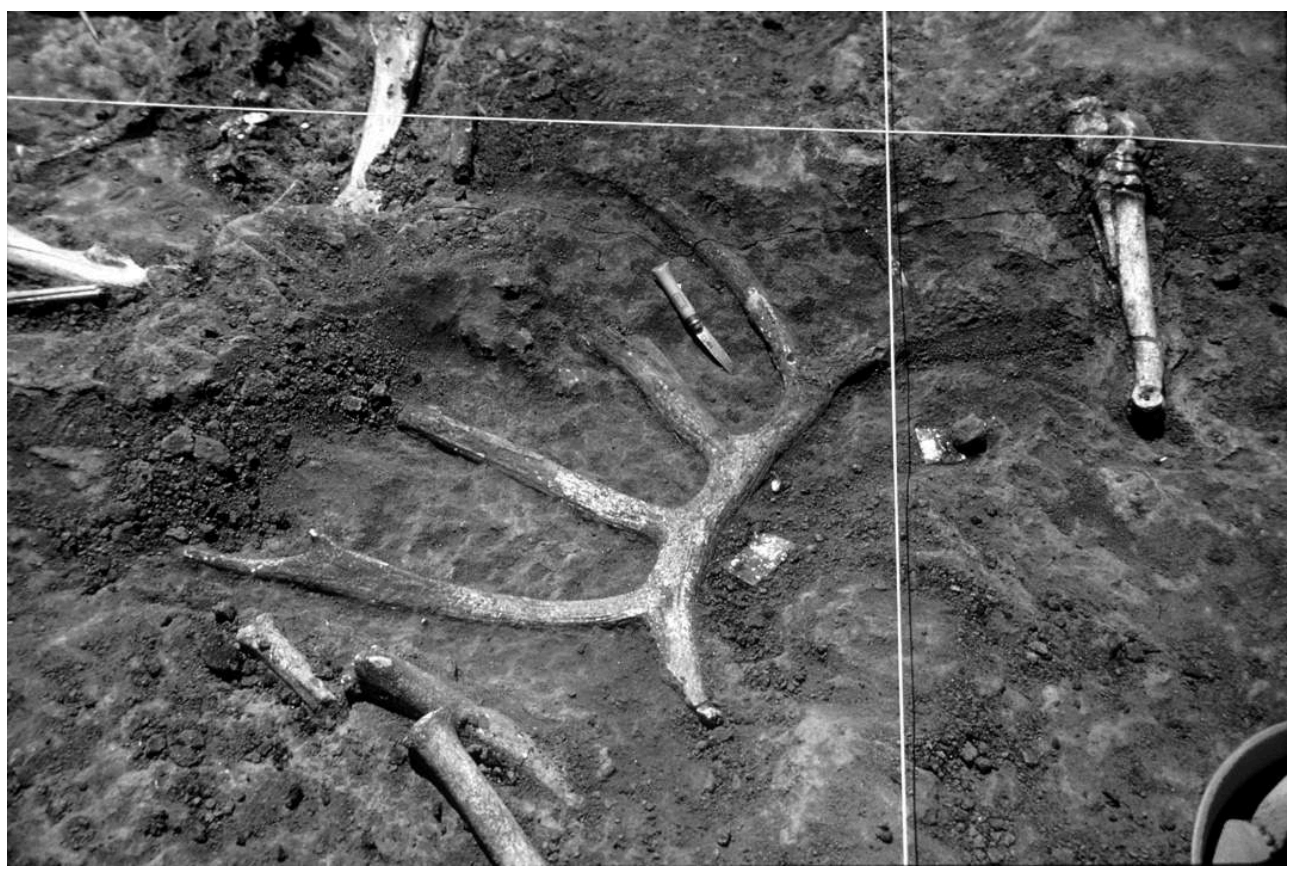

Le second andouiller est inséré en position médiane chez E. ctenoides ce qui rapproche cette espèce des grands Cerfs primitifs Praemegaceros obscurus. Les andouillers de la partie distale du bois sont pointés vers l'arrière chez E. tetraceros, alors qu'ils sont courbés plutôt vers l'avant chez le Cerf de Ceyssaguet. Heintz (1970) et de Vos et al. 
(1995) ont donné une valeur systématique à la distance entre les andouillers, or celle ci apparaît plutôt variable chez l'Eucladoceros de Ceyssaguet, ce qui suggère que ce caractère n'a pas de réelle valeur.

Les dents : Les molaires supérieures n'ont pas de cingulum, quoique l'entostylide soit bien développé. L'extrémité postérieure du protocone de la M2 et de la M3 possèdent un îlot d'émail supplémentaire et l'extrémité postérieure de l'hypocone de toutes les molaires se bifurque en forme d'éperon (fig.2).

Les prémolaires supérieures présentent un repli sur la face interne de l'hypocone ce qui fait que les prémolaires usées présentent un îlot d'émail isolé.

La mandibule est caractérisée par une branche horizontale basse et frêle si on la compare à celle de P. obscurus d'Italie (Azzarolli et Mazza, 1992); la branche montante forme avec la branche horizontale un angle de $110^{\circ}$ et le processus angulaire est bien développé. La morphologie de la $\mathrm{P} 4$ est variable ce qui rend la distinction E. ctenoides - P. obscurus difficile. Cependant les prémolaires de l'Eucladoceros de Ceyssaguet (fig.5) sont relativement plus courtes que celles de P. obscurus.

Figure 5 - Demi-mandibule droite d'Eucladoceros ctenoides (2 - 8435) : faces latérale et occlusale de la P4.

Figure 5 - Right ramus of mandible of Eucladoceros ctenoides (2 - 8435): lateral view and occlusal view of P4.

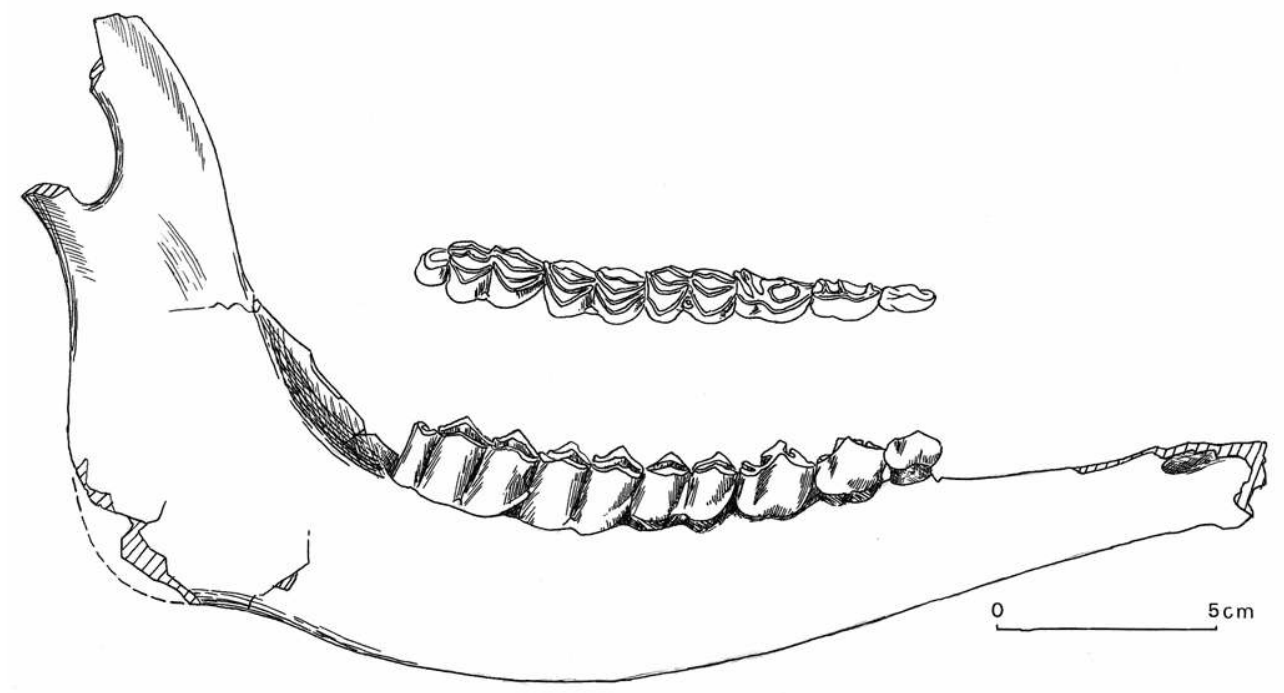

\section{2 - Genre Praemegaceros Portis | Praemegaceros obscurus Azzaroli, 1953}


vers l'arrière droit . Le premier andouiller (le "spurious tine" selon la nomenclature d'Azzaroli et Mazza, 1993) est inséré très près de la meule, il est pointé vers l'avant mais plus haut il forme un angle droit avec la perche. La base du second andouiller ("outer tine" , Azzaroli et Mazza, 1993) inséré en position médiane, se dirige aussi en position médiane. Les premier et second andouillers sont reliés par des costulations qui donnent à la perche une section transverse irrégulière qui devient subtriangulaire au dessus du second andouiller (fig.6).

Figure 6- Bois de chute de Praemegaceros obscurus (2 - 9405) : faces latérales externe (a) et interne (b).

Figure 6 - Shed antler of Praemegaceros obscurus (2 - 9405): exterior lateral view (a) and interior lateral view (b).
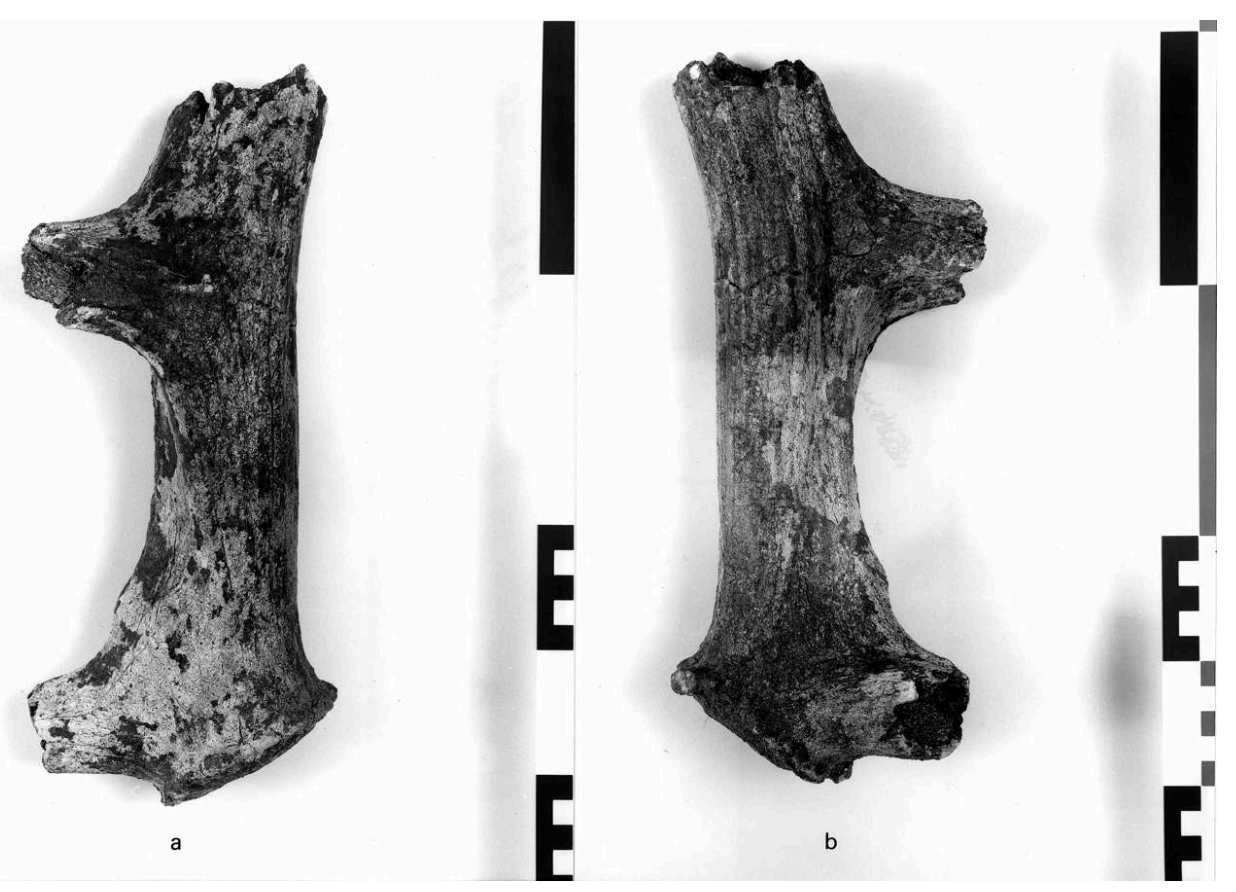

Les dents : contrairement à E. ctenoides, les prémolaires supérieures de $P$. obscurus ne présentent pas de pli d'émail sur l'hypocone et seul un pli protoconal faible est visible sur la M3 (fig.7).Les rebords labiaux du métacone et du paracone des molaires supérieures sont moins accentués que chez les Eucladoceros. L'éperon hypoconal des molaires supérieures est bien développé. 
Figure 7 - Vue occlusale de la dentition supérieure P3 - M3 (2 - 3747/5654) de Praemegaceros obscurus.

Figure 7 - Occlusion view of upper dentition P3 - M3 (2 - $3747 / 5654)$ of Praemegaceros obscurus

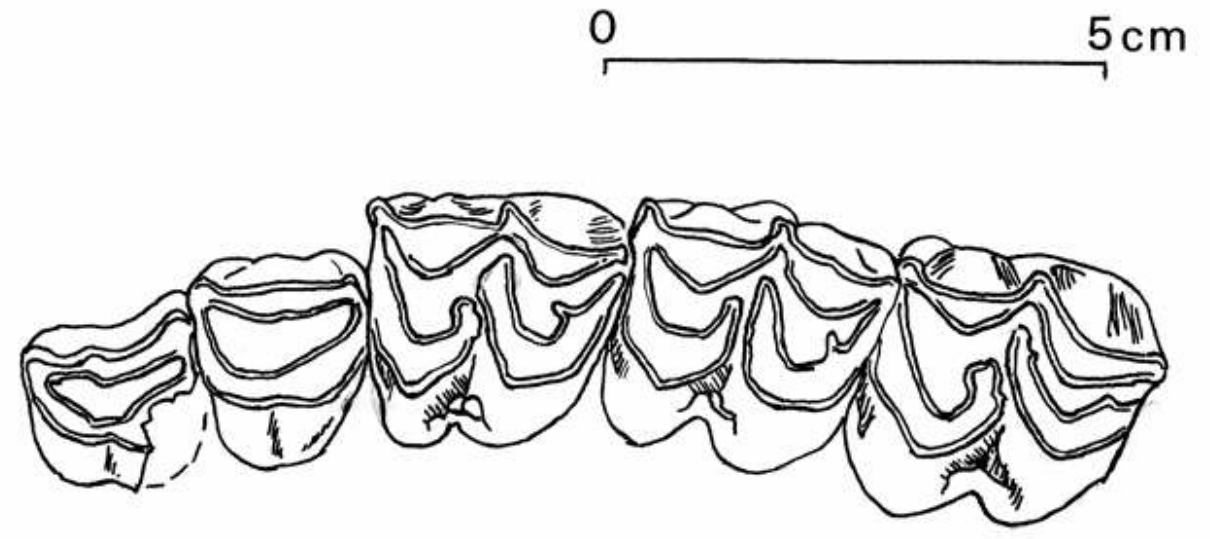

Les éléments relatifs à la mandibule sont rares et plutôt fragmentaires. La P4 est caractérisée par un degré de molarisation avancé : le métaconide s'étend vers l'avant et se connecte avec le paraconide fermant ainsi une vallée antérieure (fig. 8). A Ceyssaguet, la série des prémolaires est relativement plus longue que celle d'E. ctenoides ce qui est un caractère primitif encore présent chez P. obscurus.
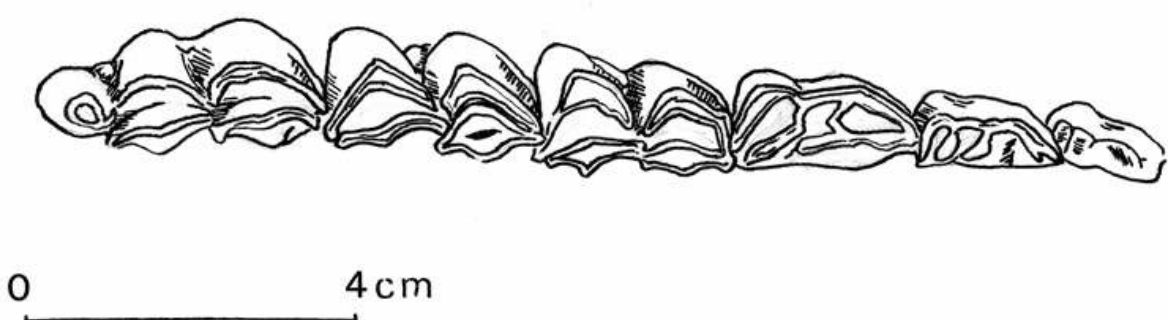

Les restes postcraniens : $P$. obscurus et E. ctenoides sont phylogénétiquement proches (Abbazzi, 1995) et la distinction des deux formes est dans certains cas difficile, ce qui fait que très peu d'ossements sont attribués à $P$. obscurus sur des critères morphologiques. On peut cependant observer qu'en dehors de la grande taille, le fémur de $P$. obscurus présente une tête courte et sphérique tandis qu'elle est étendue transversalement chez E. ctenoides (fig.9). Le col du fémur est plutôt large chez $P$. obscurus par rapport à $E$. ctenoides. Le petit trochanter est aigu et s'étend médialement chez P. obscurus alors que le même, chez E. ctenoides, forme un angle obtus et se présente de façon plus proéminente en vue caudale. L'épiphyse distale du fémur possède une dépression bien développée entre le condyle latéral et la lèvre médiane de la trochlée, ce qui n'existe pas chez E. ctenoides (fig.10). 
Figure 9 - Comparaison des fémurs de Praemegaceros obscurus $(2-10986=a)$ et Eucladoceros ctenoides $(2-12581=b)$ : importance relative de la longueur de la tête fémorale.

Figure 9 - Comparison of femora of Praemegaceros obscurus (2 - 10986=a) and Eucladoceros ctenoides ( 2 - 12581=b): importance of length of caput femoris.
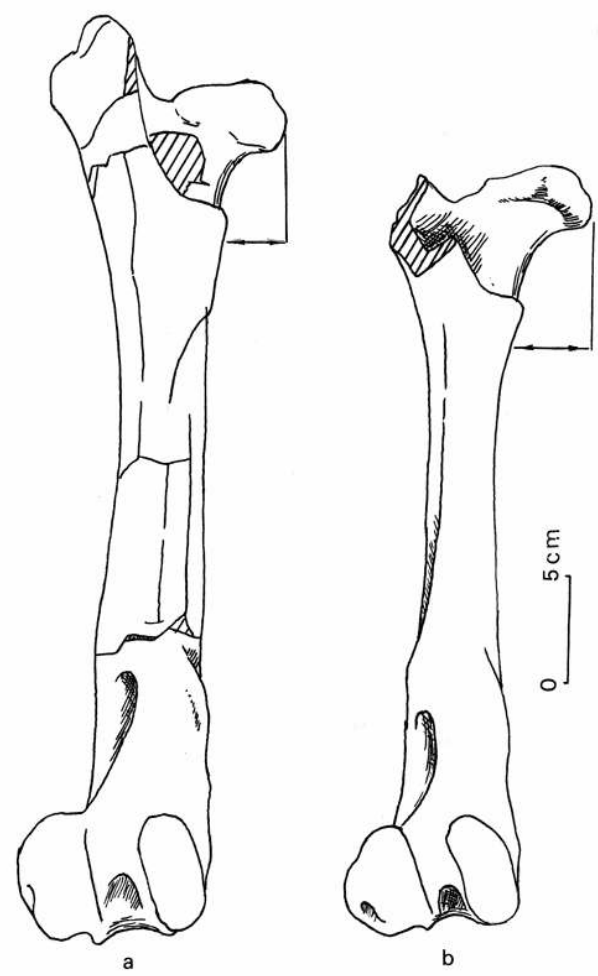

Figure 10 - Comparaison des fémurs de Praemegaceros obscurus $(2-10986=a)$ et Eucladoceros ctenoides $(2-12581=b)$.

Figure 10 - Comparison of femora of Praemegaceros obscurus (2 - 10986= a) and Eucladoceros ctenoides $(2-12581=b)$.
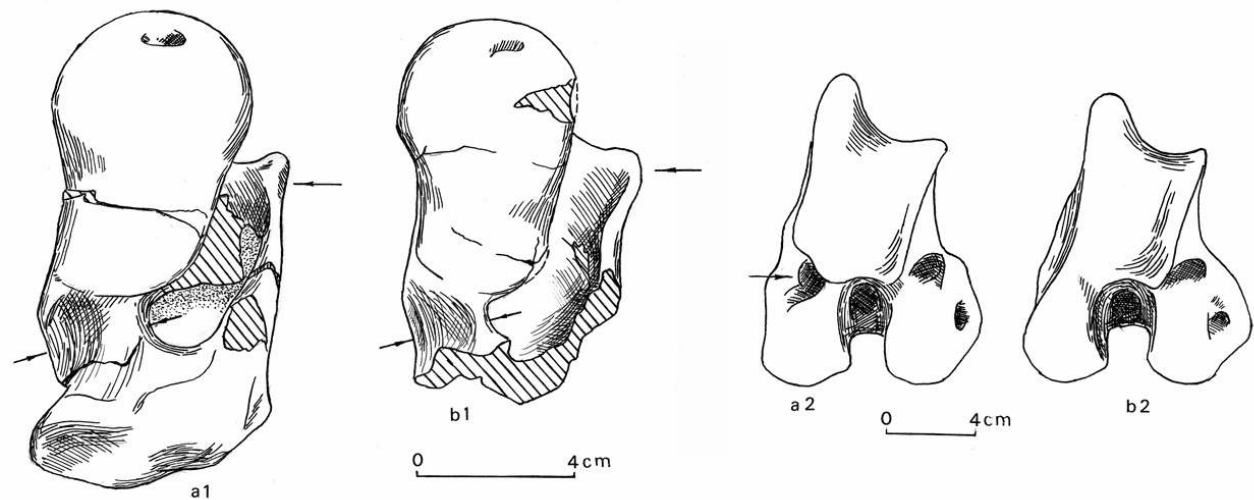

b2

- Importance relative du trochanter minor (a1 - b1)

- importance relative de la fossette située entre le condyle latéral et la lèvre médiale de la trochlée (a2 b2).

- relative importance of trochanter minor (a 1 - b1)

- relative importance of the deeping between lateral condylus and medial lip of the trochlea (a2 - b2).

Sur le tibia, l'aire intercondylaire caudale de l'épiphyse proximale d'E. ctenoides forme une palette postérieure bien développée et séparée par une profonde échancrure du condyle médial. Chez P. obscurus, cette même palette est comparativement plus faible et 
l'échancure entre palette et condyle médial peu profonde. La ligne extérieure de la crista tibiae est proche d'un triangle chez E. ctenoides, tandis qu'elle est trapézoïdale chez P. obscurus (fig. 11).

Figure 11 - Comparaison des tibias de Praemegaceros obscurus $(2-12069=a)$ et Eucladoceros ctenoides $(2-10876=b)$.

Figure 11 - Comparison of tibiae of Praemegaceros obscurus (2 - 12069=a) andEucladoceros ctenoides $(2-10876=b)$.
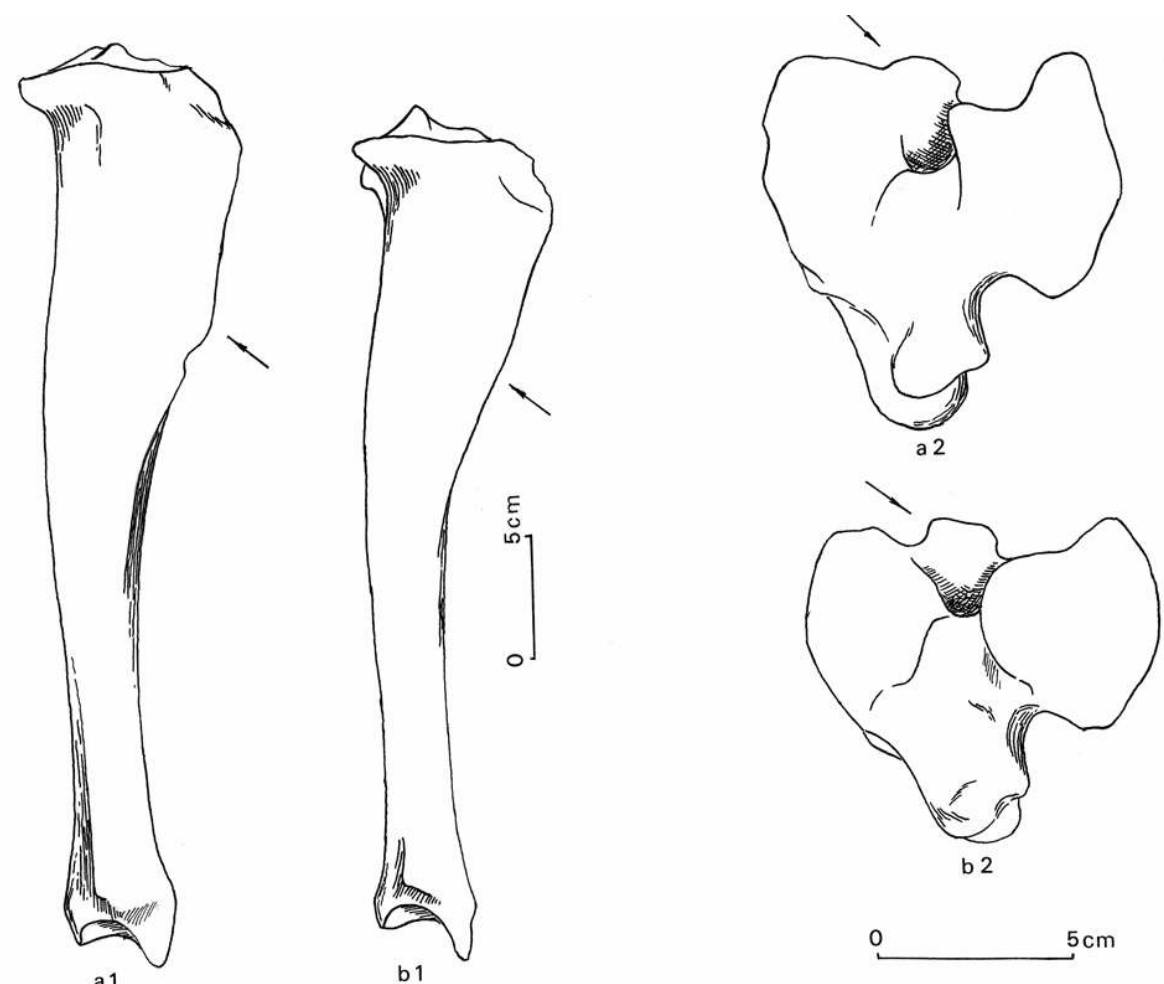

- différence de profil de la crète tibiale (a1 - b1),

- forme de l'aire intercondylaire caudale (a2 - b2).

- difference of the profil of crista tibiae

- shape of the caudal intercondylus area.

On peut ainsi rapporter les pattes en connexion de la figure 12 à E. ctenoides. 
Figure 12 - Pattes en connexion d'Eucladoceros ctenoides. Figure 12 - Articulated bones of Eucladoceros ctenoides.

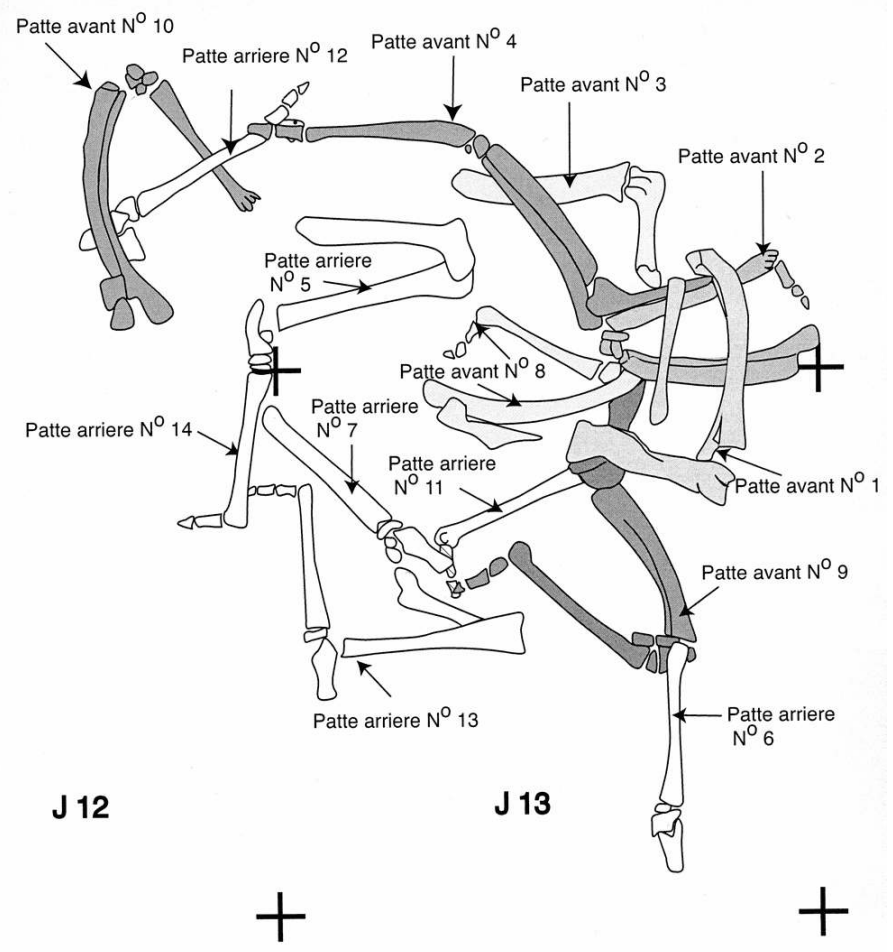

Pattes avant N. 1 : du numéro 2 - 8224 à 2 - 8232 ,

N. $2: 2-8242$ à 2 - 8256,

N.3 : 2 - 8257 à $2-8258$

N. $4: 2-8261$ à $2-8277$

N.8 : 2 - 8320 à $2-8339$

N. $9: 2-8366$ à $2-8383$

N. $10: 2$ - 8407 à 2 - 8413

Pattes arrière N. 5 : du numéro 2 - 8287 à 2 - 8289

N.6 : 2 - 8292 à 2 - 8306,

N.7: 2 - 8309 à $2-8316$

N. $11: 2$ - 8415 à $2-8425$

N.12: $2-8150$ à $2-8164$

N.13: 2 - 8169 à $2-8188$

N.14: 2 - 8346 à 2 - 8654.

forelimb N. 7: from number 2 - 8224 to 2 - 8232 ,

N.2: 2 - 8242 to $2-8256$

N.3: 2 - 8257 to $2-8258$

N.4: $2-8267$ to $2-8277$

N.8: 2 - 8320 to $2-8339$

N.9: $2-8366$ to $2-8383$

N. 10: 2 - 8407 to $2-8413$

hindlimb N.5: from number $2-8287$ to $2-8289$

N.6: 2 - 8292 to 2 - 8306

N.7: $2-8309$ to $2-8316$

N. 17: 2 - 8415 to $2-8425$

N. $12: 2-8150$ to $2-8164$

N.13: $2-8169$ to $2-8188$

N. $14: 2-8346$ to $2-8654$ 


\section{3 - Données sur la systématique des Cerfs de petite taille ("Dama- like deer")} rhenanus et son ancêtre $C$. pardinensis, forment une lignée indépendante et doivent être intégrés à un autre genre. Dietrich (1938) avait érigé en nouveau sous genre Cervus (Metacervoceros), l'espèce type Cervus pardinensis Croizet et Jobert; Samson et al. (1970) ont élevé le sous genre de Dietrich au rang de genre et c'est ce point de vue qui sera suivi dans ce travail.

Les restes se rapportant au genre Dama sont abondants dans les régions méditerranéennes au Pléistocène ancien. Cette branche phylétique est caractérisée par une morphologie évoluée du crâne et de la dentition. La boîte crânienne est très courte 
et arrondie, les pédicule sont courts et en position intérieure, les orbites et les ouvertures ethmoïdales sont larges, la P4 présente un niveau de molarisation élevé.

Parmi les plus anciennes espèces d'Europe se trouve Dama eurygonos du Villafranchien moyen terminal d'Italie.

De Lumley et al. (1998) ont décrit une nouvelle forme de Cerf de petite taille "Cervus (s.l.) nestii vallonnetensis " $n$. ssp. provenant du gisement du Vallonnet (Alpes Maritimes) : la base de bois de chute désignée comme holotype est caractérisée par un premier andouiller très robuste inséré près de la meule et formant un angle très obtus avec la perche . La morphologie de ce spécimen est visiblement différente du spécimen type de Pseudodama nestii ce qui permet de référer le Cerf du Vallonnet au genre Dama; de plus “C. nestii vallonnetensis" parait conspécifique avec Dama nestii eurygonos du villafanchien tardif de Capena en Italie (Petronio, 1979) et représenter en fait un stade phylogénétique avancé du Dama eurygonos du Val d'Arno supérieur d'Italie. Les données incomplètes sur la morphologie du Daim du Vallonnet ne permettent pas de conclure définitivement, mais nous pensons qu'il est raisonnable d'utiliser le nom sous spécifique vallonnetensis au niveau spécifique pour désigner le Daim appartenant aux faunes villafranchiennes terminales d'Europe ( = Dama vallonnetensis).

Les deux fragments d'Untermassfeld attribuées par Lumley et al. à C. nestii vallonnetensis (de Lumley et al. 1998, p. 480, fig. 15) appartiennent à Eucladoceros (fragment de bois de chute) et à Metacervoceros rhenanus ( bois de massacre).

Actuellement les relations phylogénétiques de cette espèce avec Dama clactoniana et Dama sp. du Pléistocène moyen de Tiraspol ne sont pas claires.

Le matériel de Ceyssaguet attribuable au genre de Dama est trop pauvre pour nous permettre de lui donner une attribution spécifique certaine.

\section{4 - Description anatomique des Cerfs de petite taille de Ceyssaguet}

\section{1 - Genre Metacervoceros Dietrich 1938 | Metacervoceros rhenanus Dubois, 1904}

60 Synonymies :

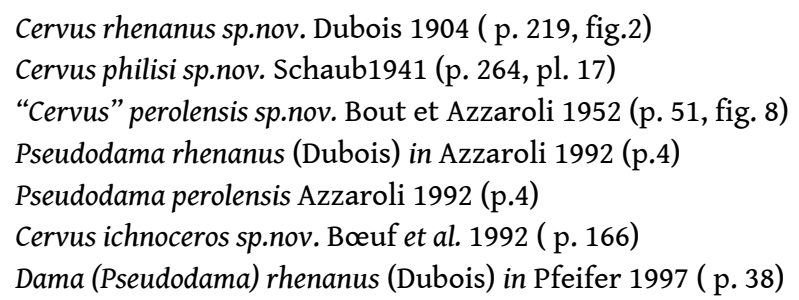

61 A Ceyssaguet, les restes de Metacervoceros rhenanus correspondent à des individus d'âge différent. Peu d'ossements ont été trouvés en connexion anatomique. Environ neuf bois sur vingt six sont des bois de massacre avec leur pédicule (35\%), dix bois de chute sur dix sept sont rongés (environ $60 \%$ ) alors que seulement deux spécimens portant le pédicule (sur neuf) sont rongés. Il est possible que les bois de massacre appartiennent à des individus morts en hiver et qu'ils aient été recouverts par la neige ce qui les a mis hors d'atteinte des Rongeurs.

Matériel étudié dans le cadre de l'étude préliminaire :

crânes complets avec les bois (2 - 2318 , 2 - 11217 et 2 - 15194),

bois de chute $(2-10564)$,

bois de massacre $(2-12292)$, 
maxillaire droit avec rangée dentaire (2 - 8398, 2 - 14603,2 - 2176),

maxillaire gauche (2 - 14393),

branche mandibulaire gauche avec P3 - M3 (2 - 2385),

radio-cubitus droit (2-10942).

Description :

63 La morphologie crânienne de $M$. rhenanus est assez particulière et diffère considérablement de celle des Cerfs de taille comparable comme Dama, Cervus, Axis et Pseudodama. Les pédicules sont longs, comprimés dorso-ventralement et tournés vers l'arrière. Les orbites sont de taille normale et leur bord antérieur atteint le niveau de la $\mathrm{M} 2$, la région orbito-frontale du crâne est courte en comparaison avec celle des Cerfs du groupe Cervus-Axis, tandis que la boite crânienne est plus longue que chez le genre Dama. Les os nasaux sont longs et atteignent la ligne de connexion du bord antérieur des orbites; les fosses préorbitaires sont très larges et profondes. L'ouverture de l'ethmoide est de taille normale. Les pariétaux et les frontaux sont convexes tandis que la surface des frontaux au dessus des orbites est concave (fig. 13 et 14).

Figure 13 - Crâne in situ de Metacervocerus rhenanus ( 2 - 15194).

Figure 13 - Cranium in situ of Metacervocerus rhenanus ( 2 - 15194).

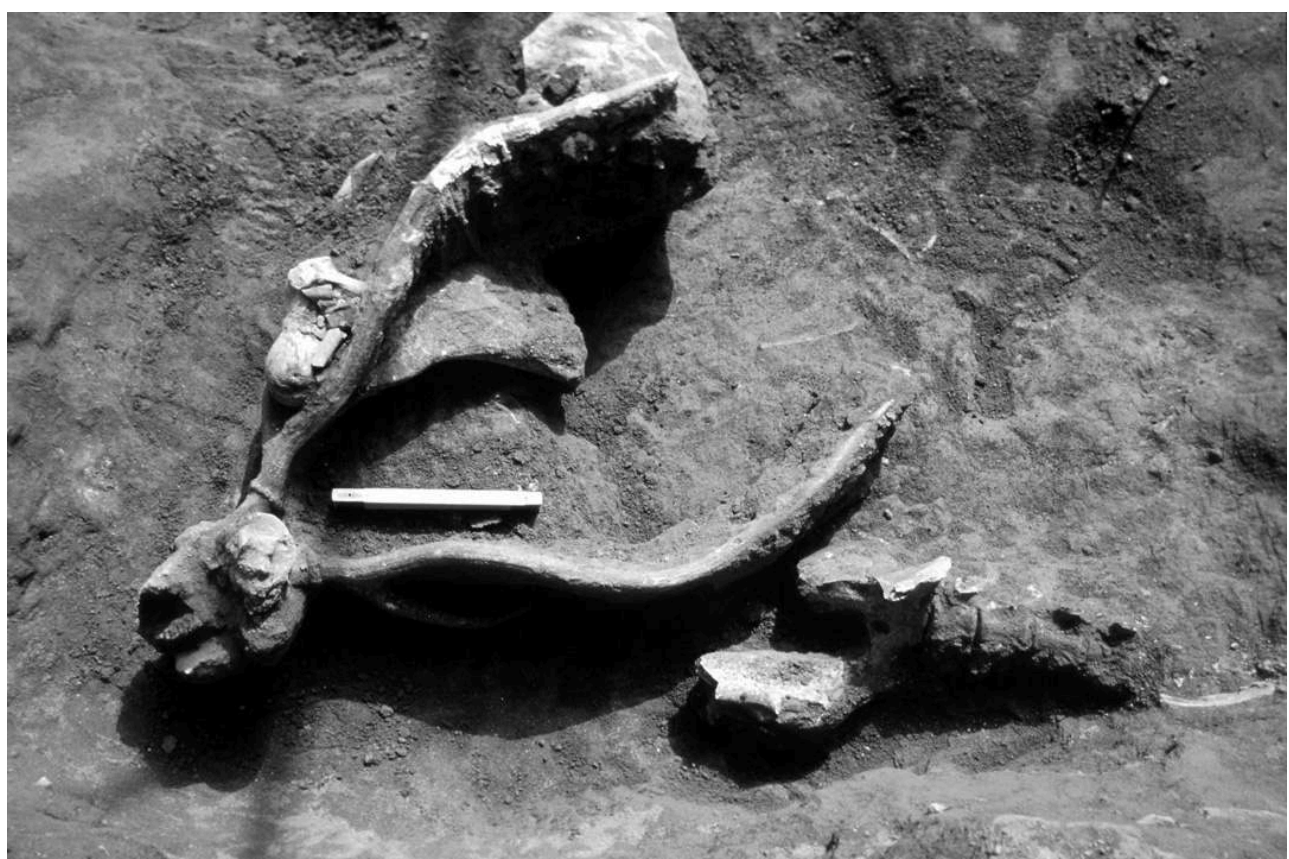


Figure 14 - Crâne de Metacervocerus rhenanus. Figure 14 - Cranium of Metacervocerus rhenanus.
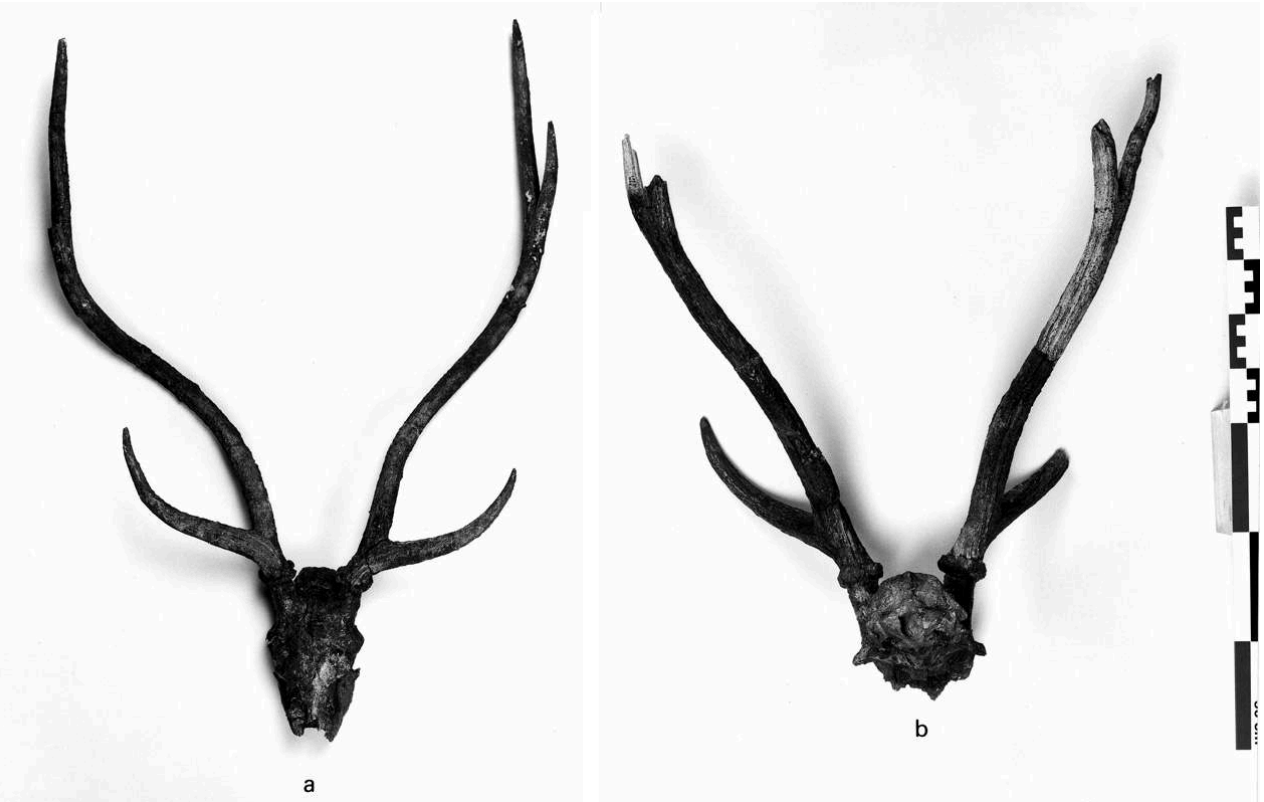

2 - 2318 : face antérieure

2 - 11217: face postérieure.

2 - 2318: antérior view

2 - 11217: posterior view.

Les caractères crâniens de $M$. rhenanus comme : le neurocrâne long, la brièveté de la région orbito-frontale ainsi que l'allongement des pédicules et leur inclinaison vers l'arrière, sont considérés comme plésiomorphiques et ils peuvent être observés sur Cervavitus du Miocène tardif (Vislobokova, 1990).

Les bois à trois pointes sont lyrés avec une section circulaire. Le premier andouiller est situé à une certaine distance de la meule et son angle de bifurcation varie de 60 à $100^{\circ}$. La surface des bois est perlée et costulée. La bifurcation distale formée par le second andouiller, qui est inséré du côté antérieur de la perche, est orientée dans un plan parasagittal (fig. 15 et 16$)$. 
Figure 15 - Bois de chute $(2$ - 10564) de Metacervocerus rhenanus. Figure 15 - Shed antler (2 - 10564) of Metacervocerus rhenanus.
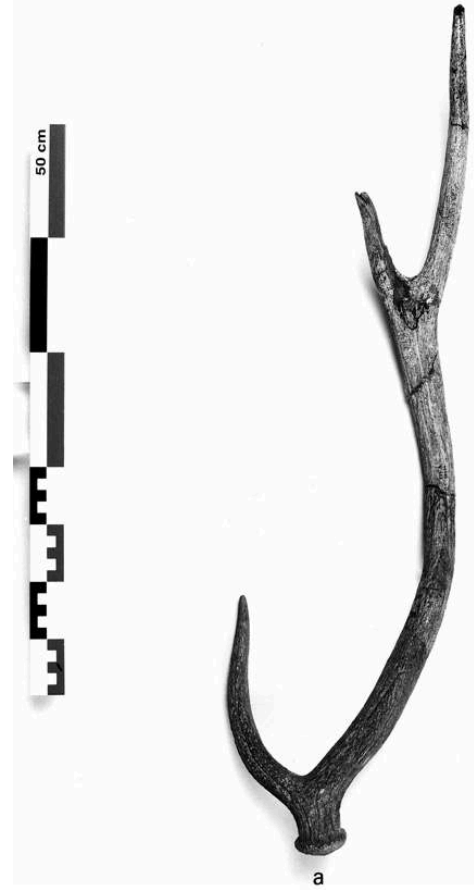

a : face externe

$b$ : face interne.

a: exterior view

b: interior view.
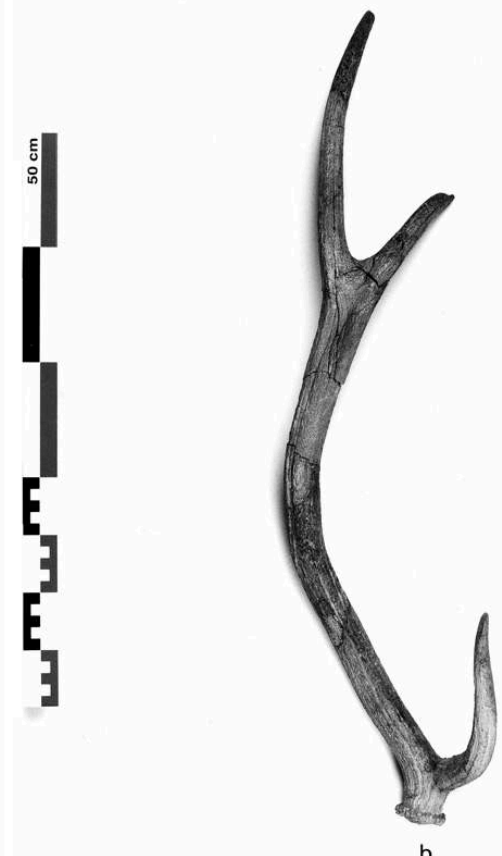
Figure 16 - Bois de massacre ( 2 - 12292) de Metacervocerus rhenanus Figure 16 - Pedicle antler (2 - 10564) of Metacervocerus rhenanus.

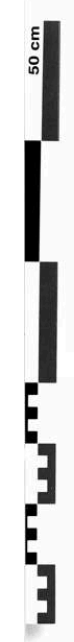

a : face externe

b : face interne.

a: exterior view

b: interior view.
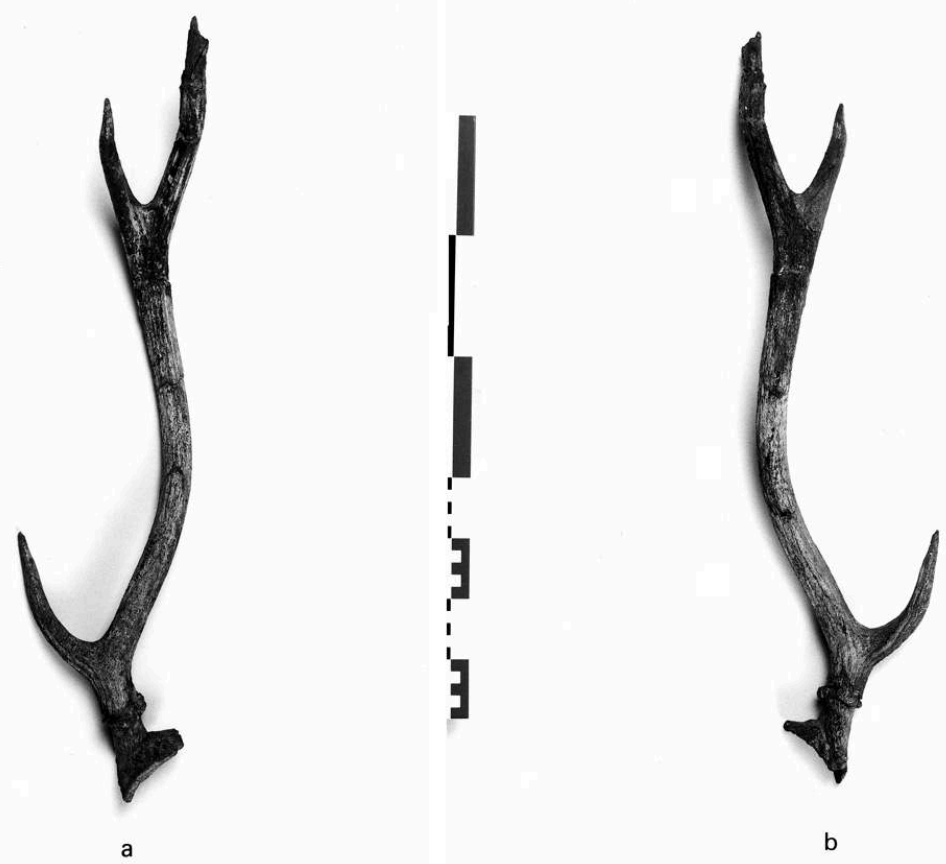

b

Contrairement aux genres Cervus et Axis, Metacervoceros possède une zone orbitofrontale courte, des orbites situés au dessus de la rangée des dents supérieures qui s'étendent au delà du bord postérieur des os nasaux, des molaires supérieures avec un cingulum plus ou moins développé. Metacervoceros se distingue du genre Dama par des pédicules longs et dirigés vers l'arrière, un neurocrane long, des ouvertures ethmoidales petites et un premier andouiller situé haut sur la perche et formant un angle aigu avec celle-ci. Les caractères énumérés ci dessus ont une valeur générique ( Vislobokova, 1990).

67 Les dents supérieures ont un cingulum effacé, un pli protoconal et un éperon. Les parastyles et mesostyles sont bien accentués et proéminents; les costulations sur la face labiale du paracone et du métacone sont très prononcées. La face interne de l'hypocone des prémolaires supérieures est très plissotée (fig. 17) ; l'angle formé par les parois linguale et labiale des molaires supérieures mesuré entre le côté labial du paracone et le côté lingual du protocone de $\mathrm{M} 1$ est de $27^{\circ}$; ce caractère est constant sur tous les exemplaires de $M$. rhenanus de Ceyssaguet et présente un intérêt systématique important. Le pli paleaomeryx manque aux molaires inférieures. La molarisation de la P4 est tout à fait primitive, cependant quelquefois le lobe postérieur du métaconide est connecté avec l'entoconide. 
Figure 17 - Dents supérieures de Metacervocerus rhenanus $(2-8398=a)$ en vue occlusive, Branche mandibulaire gauche de Metacervocerus rhenanus $(2-2385=b)$ en vue latérale et détail de la P4 (c).

Figure 17 - Upper dentition of Metacervocerus rhenanus (2 - 8398=a): occlusion view, lateral view of left mandible ramus of Metacervocerus rhenanus $(2-2385=b)$ and detail of P4 (c).

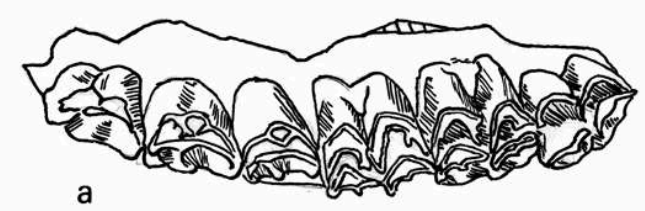

0 $4 \mathrm{~cm}$

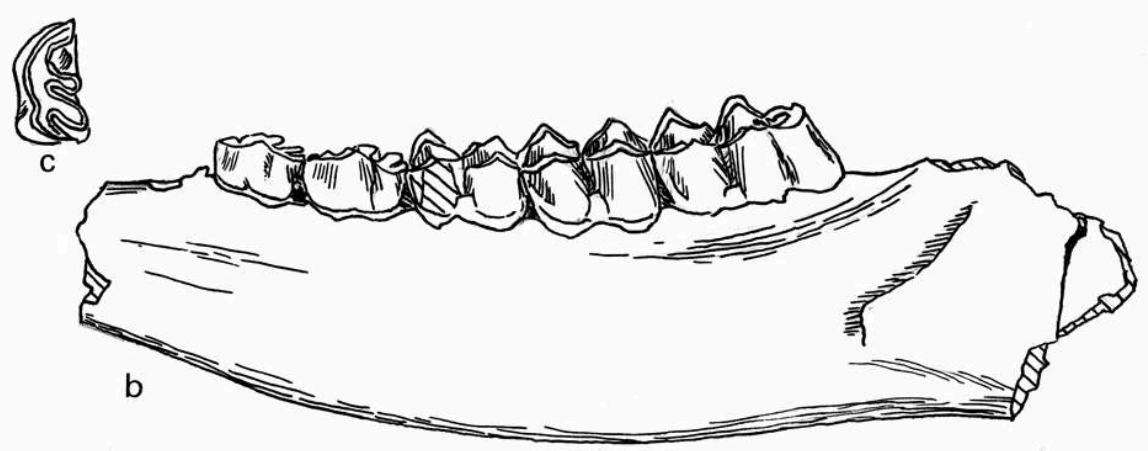

\section{2 - Genre Dama | Dama sp.}

Matériel étudié dans le cadre de l'étude préliminaire :

un maxillaire droit avec P3 - M3 (2 - 11010),

un radiocubitus gauche $(2-10332)$.

Description :

La dentition supérieure est comparativement de petite taille (fig.18). La longueur des molaires supérieures est de $44.6 \mathrm{~mm}$, alors que chez Metacervoceros rhenanus de Ceyssaguet cette valeur varie de 49.5 à $51.5 \mathrm{~mm}$. Le cingulum des molaires est réduit et l'ectostylide est très petit. Contrairement à $M$. rhenanus, l'hypocone n'a pas d'éperon, la morphologie du protocone est simple sans pli d'émail surnuméraire et, sur la face labiale des dent supérieures, le pilier des cônes et le bourrelet basilaire sont plus faibles. L'angle entre les parois labiale et linguale de la première molaire supérieure est de $35^{\circ}$. Cette valeur est très proche de celle de Dama eurygonos du Villafranchien d'Italie chez qui cet angle varie entre 37 et $38^{\circ}$. 


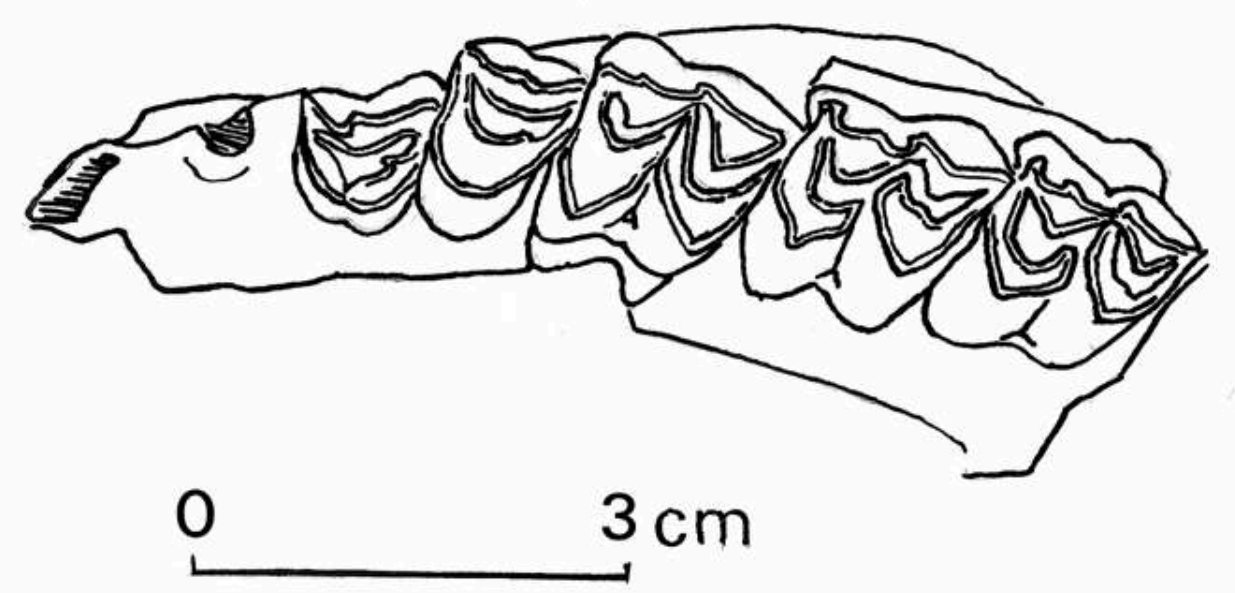

Le radiocubitus complet provient d'un cerf de petit taille qui présente des différences morphologiques avec les éléments homologues rapportés à $M$. rhenanus.. La diaphyse du radius et extrémité distale sont plus étroites ; la profondeur de la cavité articulaire de l'articulation de la partie latérale de extrémité proximale est plus réduite ce qui suggère une surface articulaire moins développée. Le processus coronoïde de l'articulation proximale est pointu et plus élevé que celui du radius de M. rhenanus. La situation du cubitus est très particulière, diffère de ce que l'on observe chez les Cerfs villafranchiens de petite taille et rapproche les éléments étudiés du Dama dama actuel: la diaphyse du cubitus est en position plus médiane par rapport à celle du radius et forme avec celle-ci un angle droit ce qui donne à l'os une construction symétrique très caractéristique. Chez $M$. rhenanus, la diaphyse du cubitus est inclinée latéralement et forme un angle aigu avec la face plantaire du radius (fig.19). 
Figure 19 - Comparaison du radiocubitus de Dama sp. $(2-10332=a)$ et de Metacervocerus rhenanus $(2-10262=b)$.

Figure 19 - Comparison of radiocubitus of Dama sp. $(2$ - 10332= a) and Metacervocerus rhenanus (2 $10942=b)$.
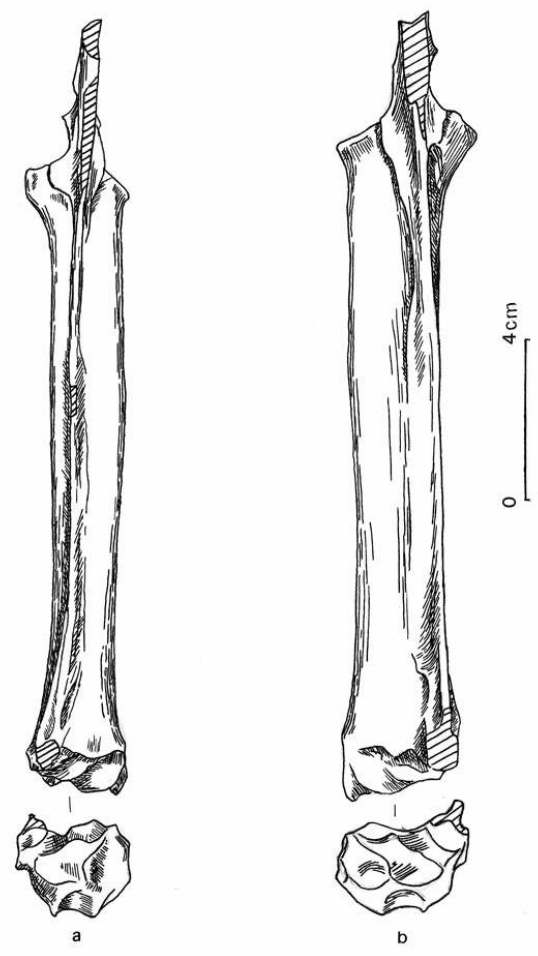

Le matériel réduit trouvé à Ceyssaguet ne comporte ni restes crâniens ni bois, aussi n'est-il pas possible d'effectuer de comparaisons directes avec Dama eurygonos ou avec le Daim du Vallonnnet, d'ou l'appellation Dama cf. vallonnetensis.

\section{Conclusions}

L'association d'un Eucladoceros aux bois simples et d'un d'un Cerf de petite taille aux bois à 3 pointes se retrouve sous des noms différents dans d'autres sites du Pléistocène ancien d' Europe de l'Ouest. Nous pouvons citer :

- de nombreux restes d'Euctenoceros tetraceros et d'autres moins nombreux de "Cervus" perolensis ont été décrits dans la faune du Creux de Peyrolles (Bout, Azzaroli, 1952),

- de même Eucladoceros ctenoides et Cervus rhenanus font partie de la faune de d'Oosterschelde en Hollande (de Vos et al. , 1995),

-Eucladoceros giulii et "Cervus nestii vallonnetensis" se rencontrent à Untermassfeld en Allemagne (Kahlke, 1997).

Il parait logique de penser que nous nous trouvons devant les mêmes formes de Cerfs qu'à Ceyssaguet : Eucladoceros ctenoides et Metacervoceros rhenanus.

74 Le schéma général de la distribution géographique de ces Cerfs provenant de sites fossilifères connus et correspondant aux faunes villafranchiennes terminales d'Europe montre que la distribution d'E. ctenoides et $M$. rhenanus correspond à la zone tempérée d'Europe de l'Ouest (fig. 20 et 22). 
Figure 20 - Aspect global de la distribution d' Eucladoceros ctenoides en Europe à la fin du Pleistocène inférieur.

Figure 20 - Global european distribution of Eucladoceros ctenoides during late inferior Pleistocene.

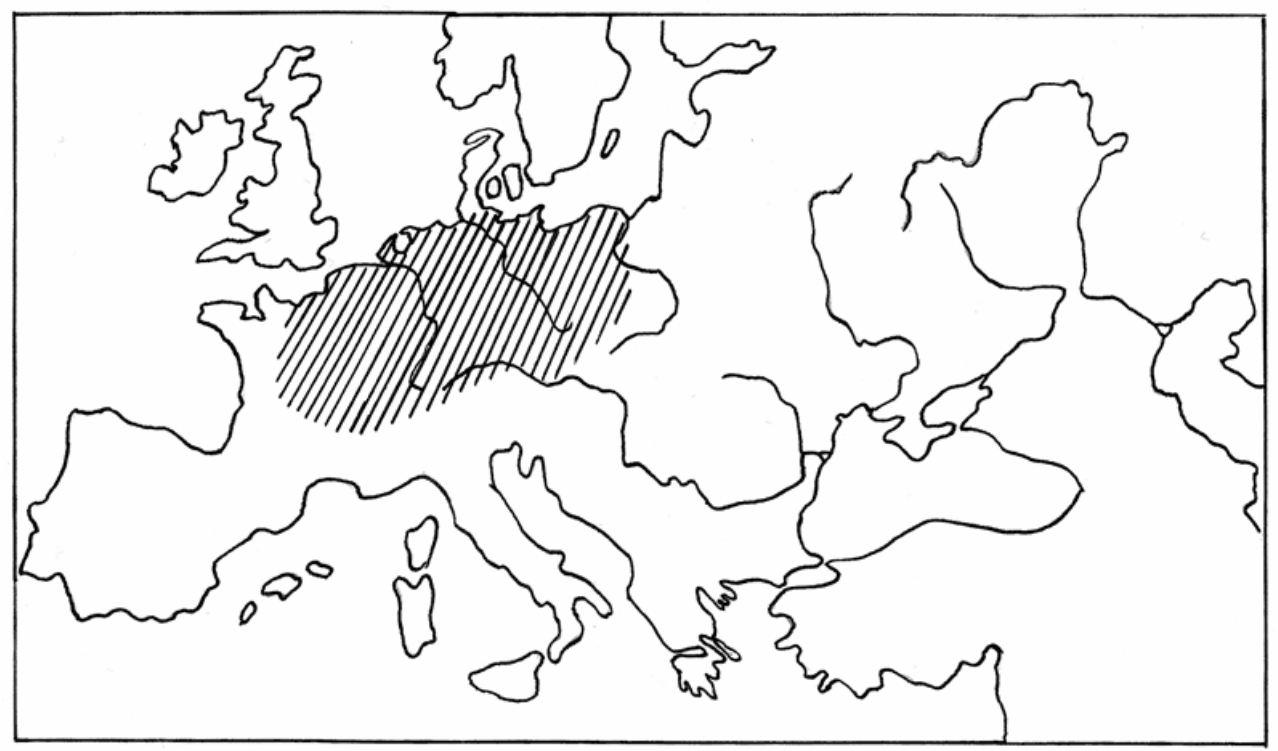

Figure 21 - Aspect global de la distribution de Praemegaceros obscurus en Europe à la fin du Pleistocène inférieur.

Figure 21 - Global european distribution of Praemegaceros obscurus during late inferior Pleistocene.

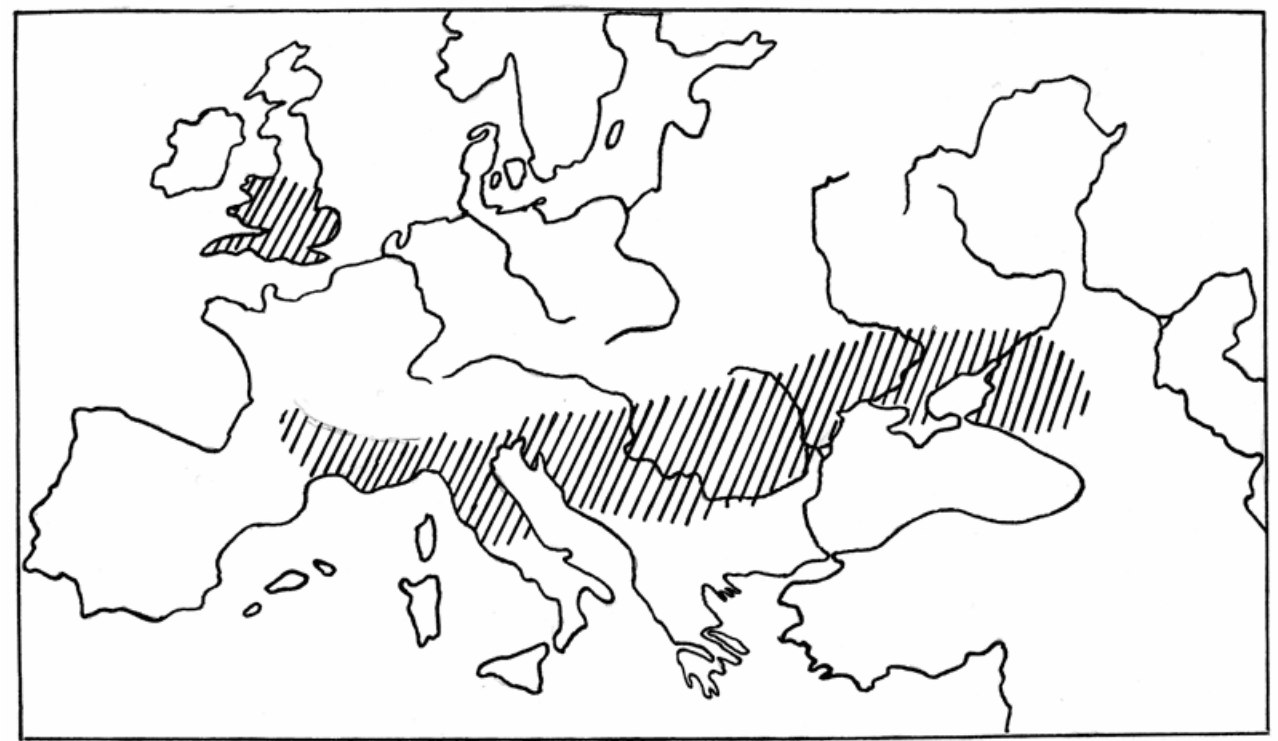


Figure 22 - Aspect global de la distribution de Metacervocerus rhenanus en Europe à la fin du Pleistocène inférieur.

Figure 22 - Global european distribution of Metacervocerus rhenanus during late inferior Pleistocene.

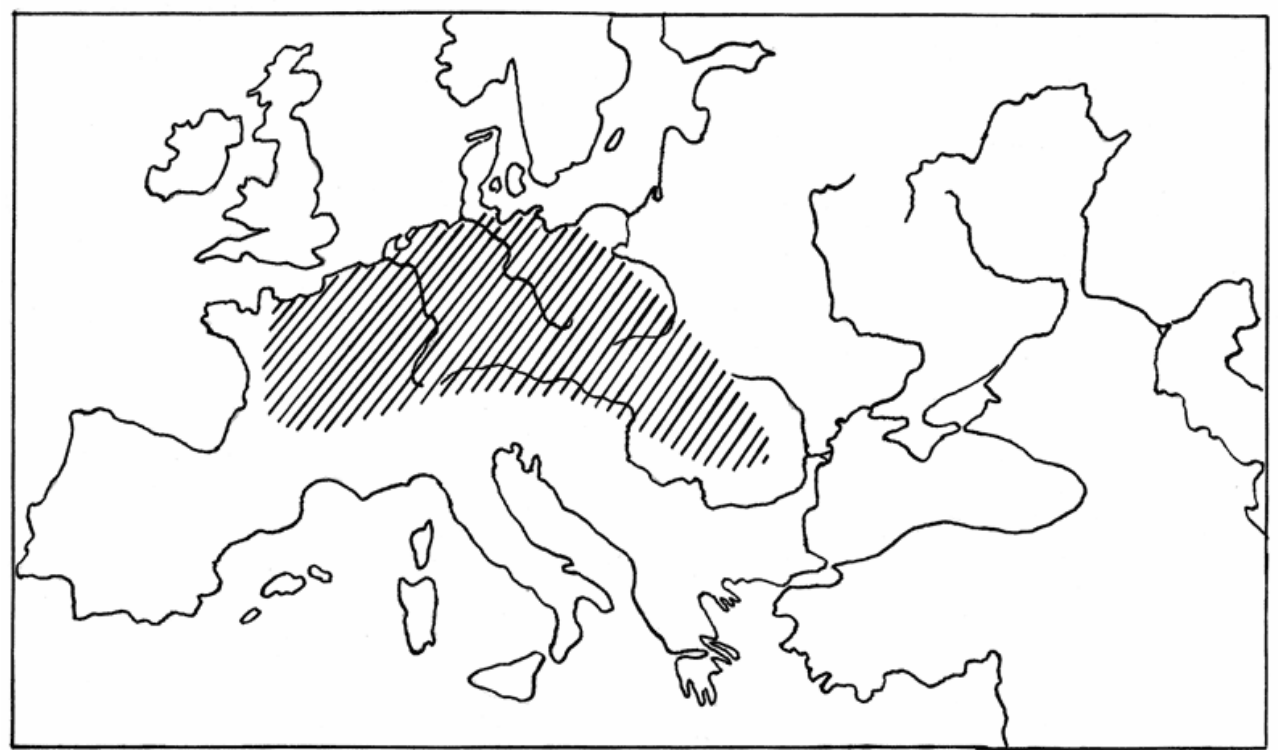

Figure 23 - Aspect global de la distribution de Dama sp. en Europe à la fin du Pleistocène inférieur. Figure 23 - Global european distribution of Dama sp. during late inferior Pleistocene.

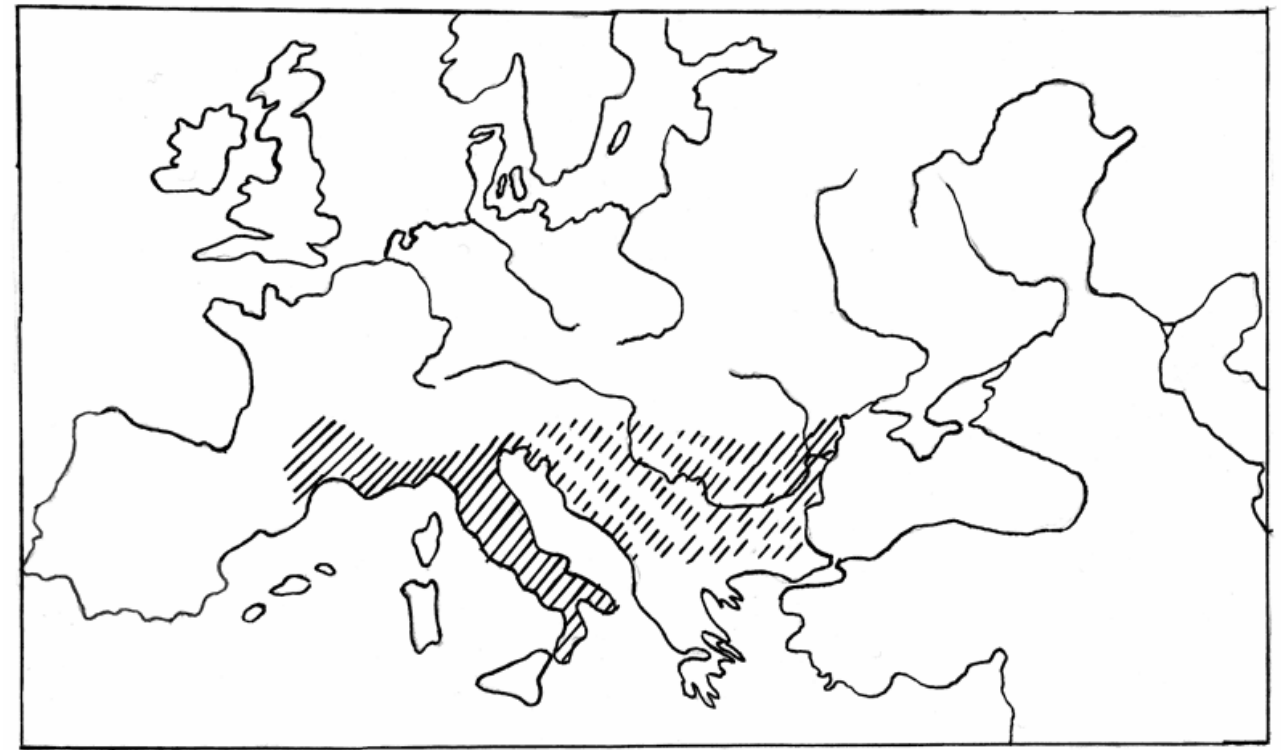

Contrairement aux deux espèces ci-dessus, la distribution de Praemegaceros obscurus correspond plutôt, à la même période, aux régions périphériques d'Europe; on le rencontre dans les faunes de Pietrafitta et Cava Liberatori en Italie centrale (Azzaroli et Mazza,1993 - Abbazzi, 1995), la formation du Cromer forest -bed en Angleterre (Azzaroli, 1953 - Lister,1993), la faune d'Odessa terminal de Salcia en Moldavie (Abbazzi et al. 1999) et à Rotbav-Silvestru en Roumanie (Radulescu et Samson, 1967).

De nombreux restes de la faune tamanienne de Semibalki, mer d'Azov, appartiennent sûrement à $P$. obscurus: ils ont été décrits par Baigusheva comme Eucladoceros aff. orientalis et P. verticornis (Baigusheva, 2000). P. obscurus est associé dans les faunes 
mentionnées ci-dessus à des cervidés de paysages ouverts comme l'Elan primitif de Semibalki et de Salcia et Dama farnetensis (=Dama eurygonos) de Cava liberatori (fig.21).

La présence d'un Daim archaïque, Dama cf. vallonnetensis dans la faune de Ceyssaguet n'est pas surprenante. Des formes de daims primitifs de type eurygonos/vallonnetensis avec des andouillers simples sans palmation sont connues dans le Pleistocène inférieur d'Italie (Azzaroli, 1947 - Petronio, 1979) et dans le sud de la Moldavie (non publié). De Lumley et al. (1998) a décrit Dama vallonnetensis associé à un grand Cerf désigné comme "Praemegaceros "s. l. n.sp. au Vallonnet.

On peut donc penser que le Daim villafranchien habitait principalement les régions européennes à climat sec et chaud (fig. 23).

Les données bibliographiques relatives à $E$. ctenoides et $M$. rhenanus permettent de penser que ces deux espèces de Cervidés peuplaient à la fin de l'histoire des faunes villafranchiennes les régions d'Europe de l'Ouest. $P$. obscurus est connu seulement à la périphérie dans les régions nord-ouest, sud et sud-est de l'Europe, tandis que le Daim archaïque, Dama de type vallonnetensis, habitait préférentiellement les zones sud de l'Europe; Il ne fait pas de doute que la distribution des Cerfs villafranchiens était conditionnée par des facteurs éco-géographiques ce qui permet de caractériser les zones climatiques villafranchiennes.

L'étude des Cerfs de Ceyssaguet permet déjà d'avancer que le site se situe dans une zone de confluence entre les cortèges fauniques ouest-européens adaptés à un climat tempéré et ceux des zones sud-européennes inféodés à des conditions plus sèches et plus chaudes.

\section{Remerciements}

Ce travail a été rendu possible grâce au Ministère de la Culture (DRAC Auvergne) et à EGIDE qui ont pris en charge les voyages et les frais de séjour de R. C. en France.

R.C. remercie particulièrement M. Georges Diener (Ambassade de France en Moldavie) pour avoir rendu possible son stage à la MMSH d'Aix-en-Provence, les membres de l'UMR 6636 pour leur aide et le Professeur Torre qui lui a facilité l'accès aux collections du Museum de Paléontologie et de Géologie de l'Université de Florence.

Les auteurs tiennent à remercier Françoise Delpech pour sa relecture attentive du manuscrit.

\section{BIBLIOGRAPHIE}

ABBAZZI L. 1995 - Megaceroides obscurus from the Val di Chiana (Cava Liberatori, Tuscany, Central Italy, f.u., early Pleistocene). Remarks on the early evolution and systematical of Megaceroides . Boll. Societa PaIeont. Ital., 34 (Z), p. 223-234. Modena.

ABBAZZI L., CROITOR R., DAVID A. 1999 - Megaceroidesobscurus (Azzaroli,1953) (Mammalia, Cervidae) from early Pleistocene sites of Eastern Moldova. Acta zool. cracov., 42 (3), p. 377 -392. Krakow.

AMBROSETTI P. 1967 - Cromerian fauna of the Rome area.Quaternaria, 9, p. 267 - 284. Rome. 
AOUADI N. 1999 - Etude préliminaire des restes crâniens de chevaux villafranchiens (Ceyssaguet,Haute-Loire). Bull. Mus. Antropol. Prehist. Monaco, 40, p. 23 - 43. Monaco.

AOUADI N., BONIFAY M.F. 1998 - Etudes paléontologique et taphonomique des restes de Proboscidiens (Ceyssaguet, Haute-Loire) Bull. Musée Anthropol. préh. Monaco, 39, p. 17 - 27. Monaco.

AZZAROLI A. 1947 - I cervi fossili della Toscana. Paleontographia Italica, XLIII, p. 46-81. Pisa.

AZZAROLI A. 1953 - The deer of the Weybourn Crag and Forest Bed of Norfolk. Bull. Brit.Mus. (Nat.Hist.), Geol., 2, 1, p. 1 - 96, London.

AZZAROLI A. 1979 - Critical remarks on some giant deer (genus Megaceros Owen) from the Pleistocene of Europe. Paleontografia Italica, 71 (n.ser. v.XLI), p. 5-16. Pisa.

AZZAROLI A. 1992. The cervid genus Pseudodama n.g in the Villafranchian of Tuscany. Paleontographia Italica, 79, p. 1-41, Pisa

AZZAROLI A., MAZZA P. 1992 - The Cervid genus Eucladoceros in the early Pleistocene of Tuscany. Paleontographia Italica, 79, p. 43-100. Pisa

AZZAROLI A., MAZZA P. 1993 - Large early Pléhistocene deer from Pietrafitta lignite mine, Central Italy . Paleontographia Italica, 80, p. 1-24. Pisa.

BAIGUSHEVA V.S. 2000 - New data on Tamanian faunal assemblage from the excavations near village Semibalki (Priazovie). ( en russe). Istoriko-archeologicheskie issledovania v Azove i na Nizhnem Donu v 1998 g., 16, p. 27-57. Azov.

BARRATA F. 1998 - Fracturations et traces au très ancien Paléolithique :étude des tibias de chevaux du gisement de Ceyssaguet (Haute Loire). Mémoire de Maîtrise de Préhistoire, Université de Provence, Aix-Marseille I.

BONIFAY M.F. 1986 - Intérêt des études taphonomiques au Pléistocène ancien : Soleihac et Ceyssaguet (Blanzac, Haute -Loire) . Bull. Mus. Hist. Nat., Paris, $4^{\circ}$ série, 8, section C, 2 , p. 269 281.Paris.

BONIFAY M.F. 1991 - Ceyssaguet (Lavoute-sur-Loire). Bilan scientifique, 1991. D.R.A. Auvergne, Ministère de la Culture Ed., 3, p. 32.Clermont-Ferrand.

BONIFAY M.F. 1995 - Apport des grandes faunes villafranchiennes du massif central français dans le cadre général du Plio-Pléistocène. $117^{\circ}$ cong. nat. soc. sav. "Vivre en moyenne montagne », p. 55 64. Editions CTHS. Paris.

BCEUF, O., GERAADS, D. ET GUTH, C. 1992 - Cervidés Villafranchiens de Blassac-la-Girondie (Haute-Loire, France). Annales de Paléontologie, 78 (3), p. 159-187. Paris.

BOULE M. 1889 - Les prédécesseurs de nos canidés. Comptes-Rendus de l'Académie des Sciences, Janvier 1889. Paris.

BOUT, P., AZZAROLI A. 1952 - Stratigraphie et Faune du creux de Peyrolles près Perrier (Puy deDome). Annales de Paléontologie, 38, p. 3-22. Paris.

DAWKINS W. B. 1878 - Contribution to the history of the European Miocene and Pliocene strata. Quat. J. Geol. Soc. London, 34, 134, p. 402 - 420. London.

DEPERET Ch., MAYET L. 1910 - Le gisement de Senèze et sa faune paleomammalogique. Assoc. Fr. Avanc. Sc., C.R. $39^{\circ}$ session Toulouse, Notes et Mémoires, II, p. 261 - 263. Paris.

DIETRICH W. O. 1938 - Zur Kenntnis der Oberpliocaenen echten Hirsche. Zeitschrift der Deutschen Geologischen Gesellschaft, 90 (5), p. 261-267. Berlin. 
DE VOS J., MOL D., J. REUMER W.F. 1995 - Early Pleistocene Cervidae (Mammalia Artyodactyla) from the Oosterschelde (the Netherlands), with a revision of the cervid genus Eucladoceros Falconer, 1868. Deinsea, 2, p. 21 - 95. Rotterdam.

DI STEFANO G., PETRONIO C. 1997 - Origin and relationships among Dama-like cervids in Europe. N.Jb.Geol. Palaeont. Abh., 207, p. 37 - 55. Stuttgart.

DUBOIS E. 1904 - On an equivalent of the Cromer Forest-bed in the Netherlands. Kon.Akad.Wetensch.Proc.Sect.Sci., 7, 3, p. 214 - 222. Amsterdam.

HADJIOUIS D. 1990 - Megaceroides algericus (Lydekker, 1890), du gisement des Phacocheres (Alger, Algerie). Etude critique de la position systématique de Megaceroides . Quatemaire, 3-4, p. 247-258. Paris.

HEINTZ E. 1970 - Les Cervidés Villafranchiens de France et d'Espagne. Memoires du Museum national d'histoire naturelle, (C) 22 , p. 1-303. Paris.

KAHLKE H.-D.1956- Die Cervidenreste aus den Altpleistozänen Ilmkiesen von Süssenborn bei Weimar. Akademien Verlag, p. 1 -3. Berlin.

KAHLKE H.-D. 1965 - Die Cerviden-Reste aus dem Tonen von Voigtstedt in Thüringen. Palaontologische Abhandlungen., A, 2, Heft 2/3, p. 381 -431, Berlin.

KAHLKE H.-D. 1997 - Die Cerviden-Reste aus dem Unterpleistozän von Untermassfeld in : Das Pleistozän von Untermassfeld bei Meiningen (Thuringen\}. Monographien des Römisch Germanischen Zentralmuseums Mainz, 40 (1), p. 181-275. Mainz.

LISTER A. M. 1993 - The stratigraphical significance of deer species in the Cromer Forest-bed Formation. Journal of Quaternary Sciences, 8 (2), p. 95 - 108.Chichester.

LUMLEY DE, H., H.D. KAHLKE, A.-M. MOIGNE, P.-E. MOULLÉ 1988- Les faunes de grands mammifères de la grotte du Vallonnet, Roquebrune-Cap-Martin. L'Anthropologie, 92, (2), p. 465-496, Paris.

PETRONIO C. 1979 - Dama nestii eurygonos Azz. di Capena (Roma). Geologica Romana, 18, p. 105-125, Roma.

PFEIFER TH. - 1997 - Dama (Pseudodama) reichenaui (Kahlke, 1996) (ArtiodactyIa : Cervidae,Cervini) aus den Mosbach-Sanden (Wiesbaden-Viebrich). Mainzer naturwiss. Archiv, 35, p. 31-59, Mainz.

RADULESCO C., SAMSON P. 1967 - Sur un nouveau cerf mégacerin du Pleistocène moyen de la dépression de Brasov (Roumanie). Geologica Romana 6, p. 317-344, Roma.

SAMSON P., RADULESCO C., KISGYÖRGY Z. 1970 - Nouvelles données sur la faune de mammifères du Villafranchien inférieur de Capeni-Virghis (dépression de Brasov,Roumanie). Eiszeitalter $u$. Gegenwart, 22, p. 64 -88. Öhringen.

SAVI F., SISMONDA A. 1841 - Sez. Geol. Min. Paleont . Atti Terz. Riun. Sci. Ital. , p. 107 - 193, Firenze.

SCHAUB S. 1941 - Die kleine Hirschart aus dem Oberpliocaen von Senèze (Hte Loire). Eclog. Geol. Helv., 34, 2, p. 264 - 271, Basel.

VAN der MADE J. 1999 - On Euraxis, on the rules of the ICZN, on methodology. N. Jb. Geol. Palaeont. Mh., (11), p. 676-678, Stuttgart.

VISLOBOKOVA I.A. 1990 - Fossil deer of Eurasia (en russe).Transactions of the Paleontological Institutte, 240, p. 1-206, Moscow. 


\section{RÉSUMÉS}

Le gisement de Ceyssaguet (Haute-Loire) a livré de nombreux fossiles dont un grand nombre de restes de Cervidés ; ceux -ci se répartissent en quatre espèces, deux de grande taille (Eucladoceros ctenoides, Praemegaceros obscurus) et deux de taille moyenne (Metacervoceros rhenanus, Dama cf vallonnetensis).

De nombreux problèmes de systématique et de synonymie sont discutés afin de comprendre la position systématique des Cerfs de Ceyssaguet.

l'occasion de cette étude, les auteurs replacent ces quatre espèces de Cervidés dans l'histoire des cervidés villafranchiens d'Europe et analysent leur distribution géographique.

The fossiliferous site Ceyssaguet ( Haute-Loire) has yielded many fossils which belong to two large-sized deer (Eucladoceros ctenoides, Praemegaceros obscurus) and two medium-sized deer (Metacervoceros rhenanus, Dama cf vallonnetensis). Numerous problems of systematics and synonymy of medium and large-sized Villafranchian deer are discussed in order to reveal the systematical position of the deer of Ceyssaguet.

An attempt is made to find a place for these four deer species in the history of Villafranchian deer of Europe and to analyse their geographical distribution.

\section{INDEX}

Mots-clés : Cervidés, faunes villafranchiennes, gisement de Ceyssaguet, Massif central français, Pléistocène inférieur

Keywords : Cervids, Ceyssaguet site, french central Massif, inferior Pleistocene, villafranchian faunas

\section{AUTEURS}

\section{ROMAN CROITOR}

Departement of Paleozoology - Institute of Zoology - Academiei str. 1 - 2028 Kishinau - Moldavie

\section{MARIE-FRANCOISE BONIFAY}

U.M.R. 6636 - MMSH - 5, rue du Château de l'Horloge - BP 647 - Aix-en-Provence Cedex 2 